\title{
Chromatin remodeling ATPase BRG1 and PTEN are synthetic lethal in prostate cancer
}

\author{
Yufeng Ding, ${ }^{1,2}$ Ni Li, ${ }^{1}$ Baijun Dong, ${ }^{2}$ Wangxin Guo, ${ }^{3}$ Hui Wei, ${ }^{3}$ Qilong Chen, ${ }^{1}$ Huairui Yuan, ${ }^{1}$ Ying Han, ${ }^{1}$ Hanwen Chang, ${ }^{1}$ Shan Kan, ${ }^{1}$ \\ Xuege Wang, ${ }^{1}$ Qiang Pan, ${ }^{1}$ Ping Wu, ${ }^{4}$ Chao Peng, ${ }^{4}$ Tong Qiu, ${ }^{5}$ Qintong Li, ${ }^{5}$ Dong Gao, ${ }^{3}$ Wei Xue, ${ }^{2}$ and Jun Qin ${ }^{1,2}$ \\ 'CAS Key Laboratory of Tissue Microenvironment and Tumor, CAS Center for Excellence in Molecular Cell Science, Shanghai Institute of Nutrition and Health Sciences, Shanghai Institutes for Biological \\ Sciences, Chinese Academy of Sciences, University of Chinese Academy of Sciences, Shanghai, China. '2Department of Urology, Renji Hospital, School of Medicine, Shanghai Jiaotong University, Shanghai, \\ China. ${ }^{3}$ State Key Laboratory of Cell Biology, CAS Key Laboratory of Systems Biology, CAS Center for Excellence in Molecular Cell Science, Shanghai Institute of Biochemistry and Cell Biology, Chinese \\ Academy of Sciences, University of Chinese Academy of Sciences, Shanghai, China. ${ }^{4}$ National Facility for Protein Science in Shanghai, Zhangjiang Lab, Shanghai, China. ${ }^{5}$ Department of Obstetrics, Gynecology \\ and Pediatrics, West China Second University Hospital, Key Laboratory of Birth Defects and Related Diseases of Women and Children, Ministry of Education, Sichuan University, Chengdu, China.
}

\begin{abstract}
Loss of phosphatase and tensin homolog (PTEN) represents one hallmark of prostate cancer (PCa). However, restoration of PTEN or inhibition of the activated PIЗK/AKT pathway has shown limited success, prompting us to identify obligate targets for disease intervention. We hypothesized that PTEN loss might expose cells to unique epigenetic vulnerabilities. Here, we identified a synthetic lethal relationship between PTEN and Brahma-related gene 1 (BRC1), an ATPase subunit of the SWI/SNF chromatin remodeling complex. Higher BRG1 expression in tumors with low PTEN expression was associated with a worse clinical outcome. Genetically engineered mice (CEMs) and organoid assays confirmed that ablation of PTEN sensitized the cells to BRG1 depletion. Mechanistically, PTEN loss stabilized BRG1 protein through the inhibition of the AKT/ CSK3ß/FBXW7 axis. Increased BRG1 expression in PTEN-deficient PCa cells led to chromatin remodeling into configurations that drove a protumorigenic transcriptome, causing cells to become further addicted to BRC1. Furthermore, we showed in preclinical models that BRG1 antagonist selectively inhibited the progression of PTEN-deficient prostate tumors. Together, our results highlight the synthetic lethal relationship between PTEN and BRG1 and support targeting BRG1 as an effective approach to the treatment of PTEN-deficient PCa.
\end{abstract}

\section{Introduction}

Prostate cancer (PCa), a leading cause of cancer mortality in males worldwide, develops through a stepwise process involving prostatic intraepithelial neoplasia (PIN), adenocarcinoma, and metastatic cancer (1). Genetic and epigenetic alterations, including but not limited to phosphatase and tensin homolog (PTEN) deletion, TMPRSS2-ERG translocation, SPOP mutation, and Myc amplification, facilitate disease progression (2-5). Loss of PTEN and hyperactivation of PI3K/AKT signaling are recognized as tumor drivers pathologically linked to $\mathrm{PCa}(2,3,6,7)$. Approximately $30 \%$ of primary tumors and up to $70 \%$ of metastatic cancers exhibit loss of heterozygosity at the PTEN gene locus $(2,3)$. Similarly, genetically engineered mice (GEMs) have revealed a key role of PTEN signaling in PCa $(8,9)$. Prostate-specific deletion of Pten $\left(\right.$ Pten $^{\mathrm{PC}-/-}$ ) results in high-grade PIN (HGPIN), with nearly $100 \%$ penetrance, which potently develops into prostatic adenocarcinoma after a long latency (8). Furthermore, Pten loss functionally cooperates with other signal alterations, such as deletion of Tp53 or Nkx3.1 and overexpression of ERG or Kras ${ }^{\mathrm{G} 12 \mathrm{D}}$, to produce full-

Authorship note: YD, NL, and BD contributed equally to this work. Conflict of interest: The authors have declared that no conflict of interest exists. License: Copyright 2019, American Society for Clinical Investigation.

Submitted: July 13, 2018; Accepted: November 20, 2018

Reference information: J Clin Invest. 2019;129(2):759-773.

https://doi.org/10.1172/JCl123557. blown disease in mice (10-14). Together, these results highlight the functional importance of PTEN in prostate tumorigenesis. Nevertheless, pharmacological targeting of PTEN or the PI3K/ AKT pathway remains a major hurdle in disease intervention.

Loss of tumor suppressor genes frequently renders cells dependent on genes otherwise not required in normal cells, a phenomenon termed synthetical lethality (15). One prominent example is the interaction between the BRCA1/2 and PARP genes that has been harnessed to develop successful cancer therapy $(16,17)$. Alterations in epigenetic machinery, including mutation or overexpression of chromatin remodelers and modifiers as well as DNA hypermethylation, regulate the plasticity of tumor cells (18). In line with this notion, studies have indicated that loss of tumor suppressors or gain of oncogenes alters the epigenetic landscape of cancer cells to confer growth advantage, but meanwhile exposes them to unique epigenetic vulnerabilities (18-20). Thus, targeting epigenetic vulnerabilities, such as ARID1A/EZH2, ARID1A/ARID1B, and PTEN/CHD1 interactions, leads to synthetic lethality in tumors under specific genetic contexts (21-24). For example, the inhibition of EZH2 selectively suppresses the growth of ovarian cancer cells containing loss-of-function variants of $\operatorname{ARID1A}(21,22)$. Recent studies showed that CHD1 is an essential downstream effector of PTEN that activates TNF- $\alpha / N F-\kappa B$ signaling in PCa (24).

Large-scale cancer genome profiling studies have identified frequent mutations in various subunits of the mammalian SWI/ SNF chromatin remodeling complex (25-27), which consists of 
A

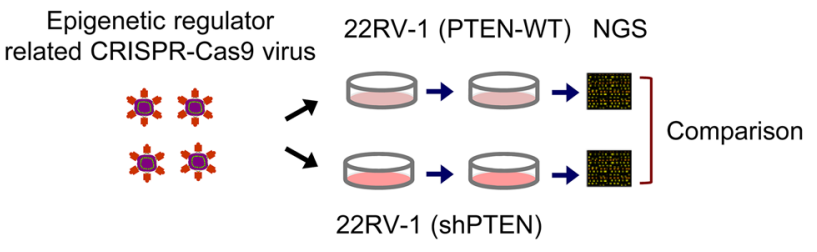

D

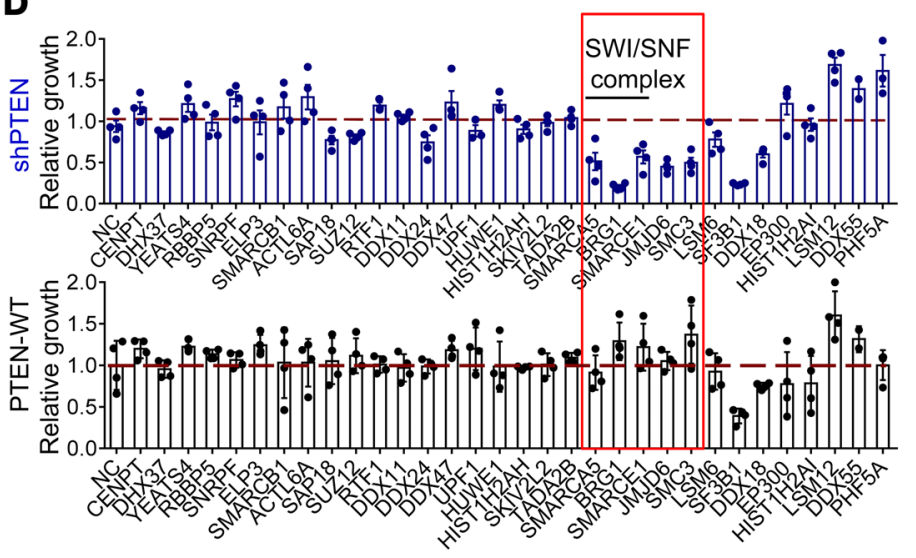

$\mathbf{F}$
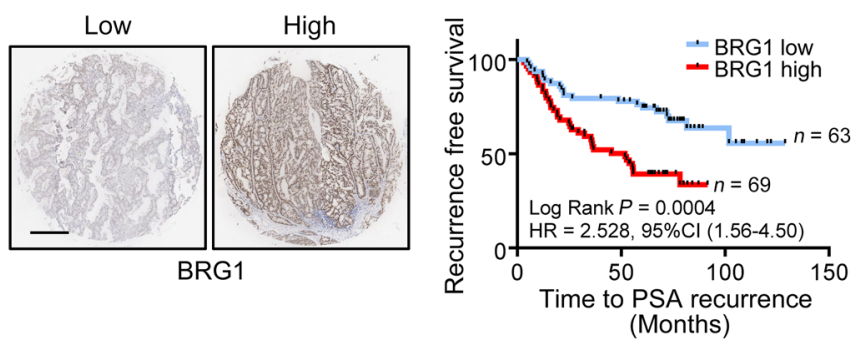

B

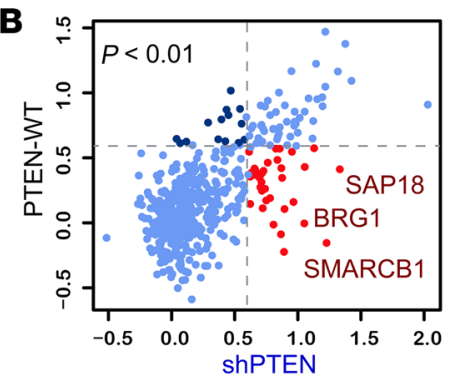

$\mathbf{E}$

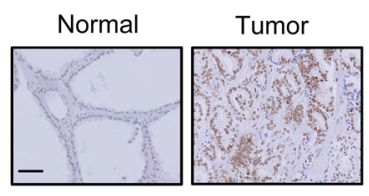

C

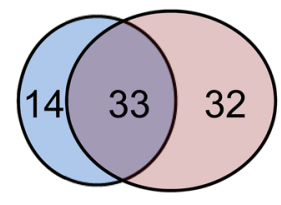

PTEN-WT ShPTEN
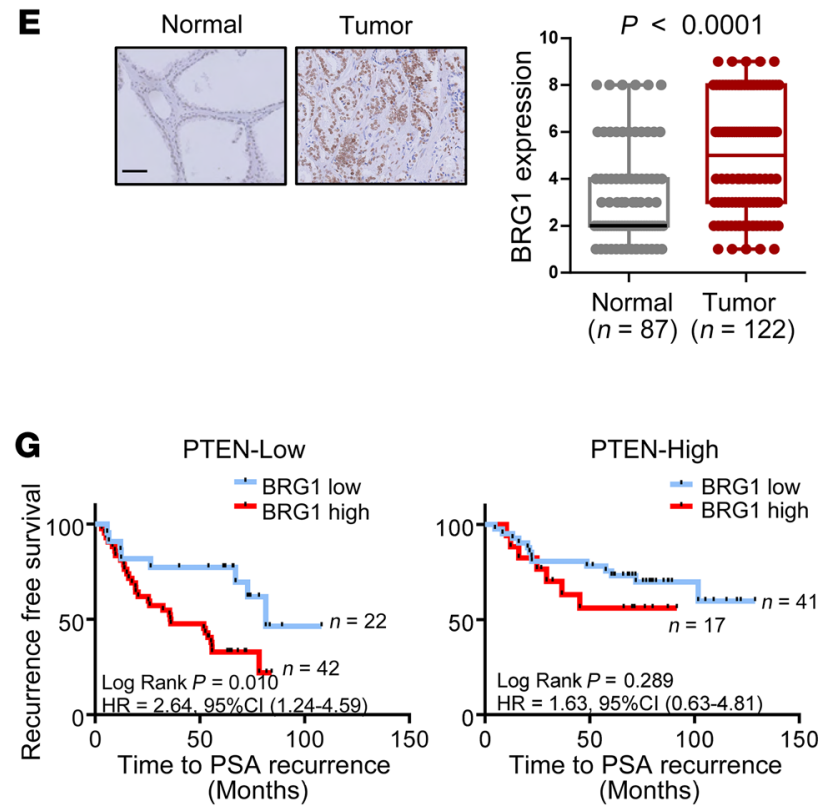

Figure 1. Identification of the epigenetic regulator required for PTEN-deficient PCa cells. (A) A schematic of the screening workflow for the chromatin regulator based on CRISPR-Cas9 screening in PTEN-WT and PTEN-KD (shPTEN) 22RV-1 cells. (B) Scatter plot showing the normalized counts for each sgRNA in the original pool (day 0 ) relative to the samples taken after 45 days of cultures (MaGeCK and MAGeCK-VISPR analysis). (C) Venn diagram showing the number of overlapping genes between the cells as indicated. (D) siRNA KD of 32 candidate genes and their effects on the growth of PTENWT and PTEN-KD 22RV-1 cells. Quantitative results shown are representative of 4 experiments. Genes highlighted in red box exhibited the differential growth effects between PTEN-WT and PTEN-KD 22RV-1 cells. (E) BRG1 staining indexes using a 10-point quantification scale in cohorts of normal prostate tissue $(n=87)$ and prostate tumors $(n=122)$ (Wilcoxon's rank sum test). Scale bar: $50 \mu \mathrm{m}$. (F) Kaplan-Meier plot of recurrence after radical prostatectomy based on the BRG1 expression index in patients ( $P$ values by log-rank test). Scale bar: $200 \mu$ m. (G) Kaplan-Meier plots based on BRG1 expression in PTEN-low and PTEN-high tumors (log-rank test).

mutually exclusive Brahma-related gene 1 (BRG1; SMARCA4) or Brahma (BRM; SMARCA2) ATPase subunits. The SWI/SNF chromatin remodeling complex functions in mobilizing nucleosomes and higher-order chromosome dynamics to regulate gene transcription (28). Despite its initially reported tumor suppressor role in cancer, BRG1 is also known to promote tumorigenesis in pancreatic cancer and colorectal cancer (29-31). Thus, BRG1 has an important role in tumorigenesis, not only when downregulated, but also when overexpressed, depending on the cellular and genetic milieu. Nevertheless, the implication and therapeutic potential of BRG1 in PCa remain largely unknown.

Here, we identified a synthetic lethal relationship between PTEN and BRG1 in an unbiased screen. Mechanistically, PTEN loss stabilized BRG1 protein through inhibition of the AKT/ GSK3 $\beta$ /FBXW7-mediated ubiquitin proteasome pathway. Increased BRG1 expression led to chromatin remodeling and initiated a protumorigenic transcriptome. Importantly, we show that BRG1 inhibition is a promising approach against PTENmutant prostate tumors in preclinical models.

\section{Results}

Screen of chromatin modifiers identifying BRG1 is required for PTEN-deficient PCa cell growth. Given the recent success in targeting chromatin regulators in cancer, we performed a CRISPRCas9-based screen in 22RV-1 cells with or without PTEN knockdown (PTEN-KD or shPTEN) to identify epigenetic regulators specifically required in PTEN-deficient PCa cells. The library consists of 517 genes encoding epigenetic writers, erasers, and readers (at least 4 sgRNAs per gene; Supplemental Table 1; supplemental material available online with this article; https://doi.org/10.1172/ JCI123557DS1). Cells were continuously passaged for approximately 45 days, and the abundance of each sgRNA was scored by calculating the $\beta$ score-based fold change relative to their initial representation (Figure 1A). The primary screen identified 47 and 
A
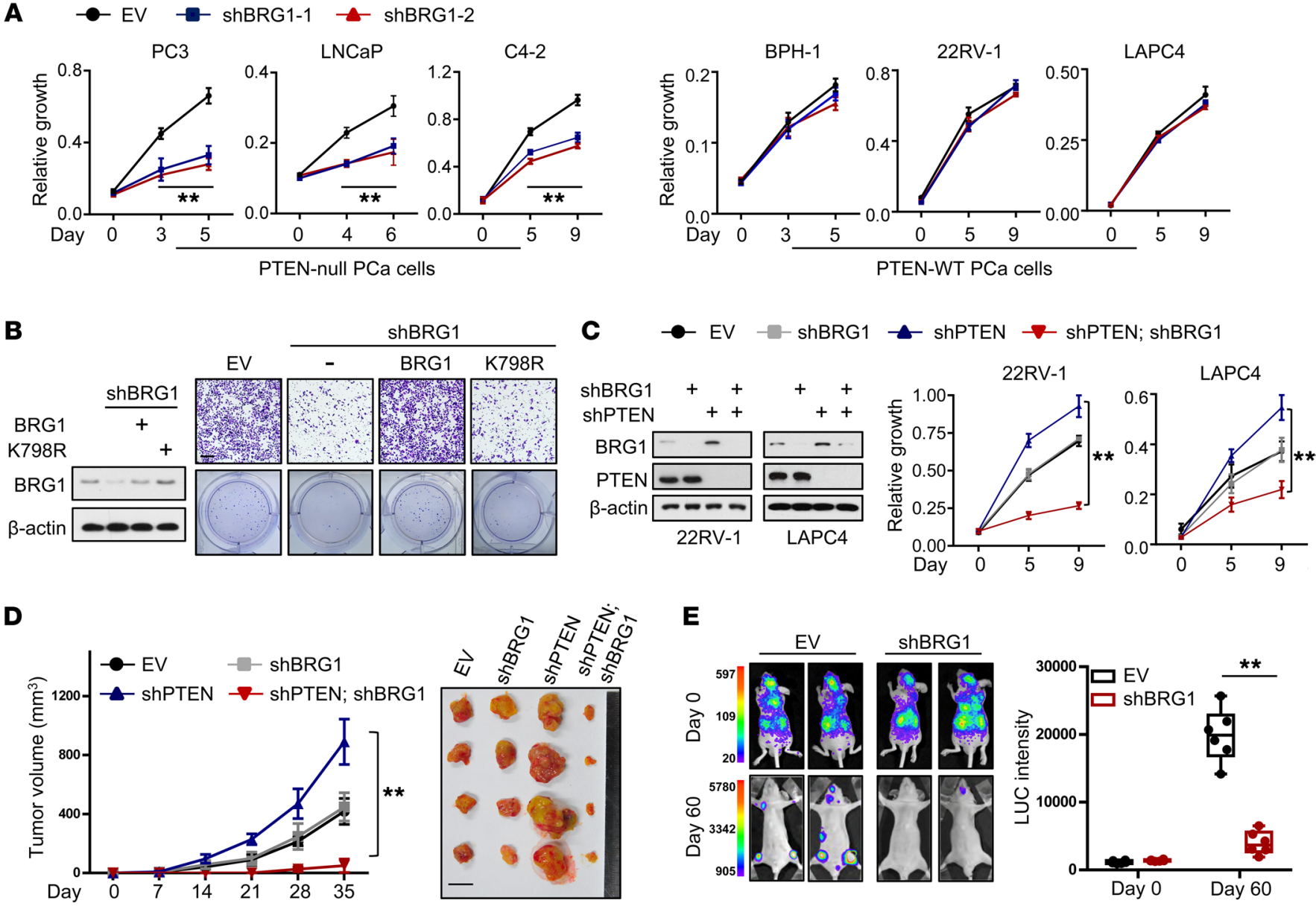

E
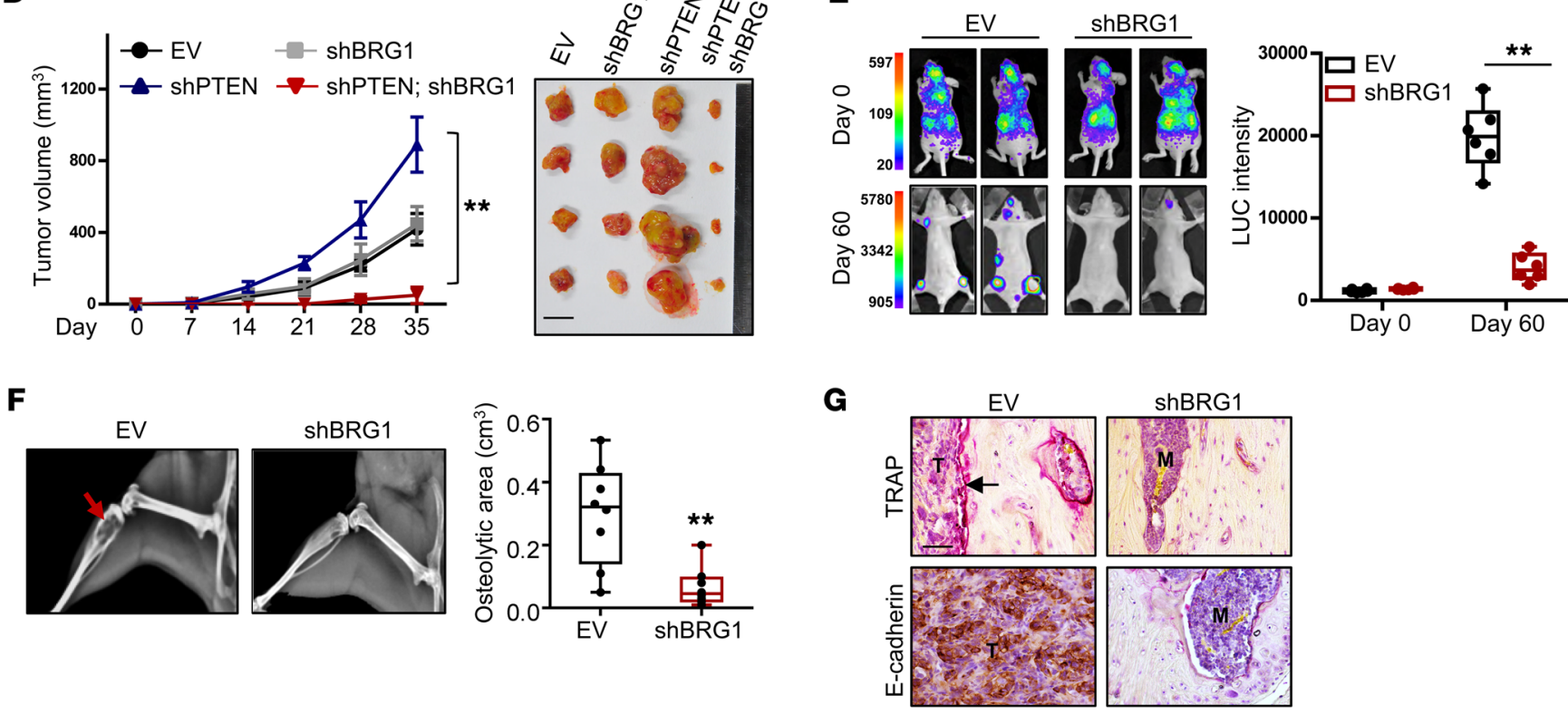

Figure 2. BRG1 is required in PTEN-deficient PCa cells. (A) MTT analysis of PCa cells with or without BRG1 KD (shBRG1). (B) Transwell (upper right) and soft agar (lower right) images of BRG1-KD PC3 cells with or without WT or mutant BRG1 (K798R) restoration. Scale bar: 1 mm. (C) IB of lysates and cell growth measurements in control and BRG1-KD 22RV-1 and LAPC4 cells with or without PTEN KD (shPTEN). (D) Measurement of subcutaneous tumor growth of control and PTEN-KD 22RV-1 cells with or without BRG1 depletion (shBRG1) ( $n=6$, 2-way ANOVA followed by Tukey's multiple comparisons test); a representative image is shown. Scale bar: $1 \mathrm{~cm}$. (E) Representative BLI images for control and BRG1-KD PC3 cells at day 0 (upper panels) and day 60 (lower panels). Limb metastasis is calculated as the mean \pm SEM of the bioluminescence signal at day 60 ( $n=6$ per group, 2-tailed Student's $t$ test). (F) Representative $x$-ray images of bone metastasis are shown on the left, and the osteolytic area is quantified on the right ( $n=8$, 2-tailed Student's $t$ test). (C) TRAP- and E-cadherin-stained images as indicated. T, tumor cell; M, bone marrow; arrow, TRAP-positive cell. Scale bar: $50 \mu \mathrm{m}$. Data represent mean \pm SEM of 3 independent experiments. Statistical analyses were performed by 2 -way ANOVA followed by Tukey's multiple comparisons test $(\mathbf{A}$ and $\mathbf{C})$. ${ }^{* *} P<0.01$.

65 gene-targeting sgRNAs with decreased abundance in PTENintact and PTEN-KD cells, respectively (Figure 1B), and the genes are summarized in Supplemental Figure 1A. As indicated by the Venn diagram, 33 genes overlapped in both cell types (Figure 1C), suggesting that they might be important for the growth of $\mathrm{PCa}$ cells regardless of PTEN level. Next, to identify epigenetic regulators important for PTEN-deficient tumors, we focused on another 32 genes and performed a validation screen using individual siR-
NAs to compare their effects in 22RV-1 cells with or without PTEN knockdown. As shown by MTT assay, BRG1 was the most potent hit (Figure 1D). BRG1 ablation clearly led to the expected difference between PTEN-WT and PTEN-KD cells (Figure 1D). In contrast, genes such as SF3B1 and DDX18 did not exhibit this selectivity. Interestingly, multiple SWI/SNF constituents (e.g., SMARCE1/ BAF57 and SMARCA5/SNF2H) were enriched in PTEN-KD cells (Supplemental Figure 1A), indicating that the SWI/SNF complex 
might confer a growth advantage to these cells. Therefore, BRG1 was chosen for further analysis, as it is the enzymatic subunit of the SWI/SNF chromatin remodeling complex and can be modulated by small molecule inhibitors.

BRG1 expression correlates with poor outcome in PTEN-low PCa patients. To evaluate the clinical relevance of BRG1 in PCa, we performed IHC with a prevalidated antibody against BRG1 of an Asian radical prostatectomy tissue microarray (TMA) composed of 122 specimens $(32,33)$. Examination of prostate specimens showed higher BRG1 expression in tumors (mean $=4.8 ; n=122$ ) than in normal tissues (mean $=3.2 ; n=87$, Figure $1 \mathrm{E}$ ). The BRG1 immunostaining intensity tended to positively associate with the Gleason score and PSA levels in tumors (Supplemental Figure 1B). Patients with elevated BRG1 levels exhibited a higher risk of biochemical recurrence $(P=0.0004$; Figure $1 \mathrm{~F})$. We further stratified patients based on PTEN levels. Kaplan-Meier survival estimate analyses revealed that BRG1 protein level was positively associated with worse prognosis in tumors with low PTEN expression $(P=0.010$; Figure $1 G)$. In contrast, the prognostic significance of BRG1 did not reach statistical significance in tumors with high PTEN expression $(P=0.289$; Figure $1 G)$. These results suggest a causal role of BRG1 in prostate tumorigenesis in the context of PTEN deficiency.

BRG1 ablation shows synthetic lethality in PTEN-deficient $P C a$ cells. To determine whether BRG1 is specifically required in PTEN-deficient PCa cells, we first investigated BRG1 functions in a panel of PCa cell lines. Using 2 different shRNA constructs to deplete BRG1 expression (Supplemental Figure 2A), we found that reduced $B R G 1$ expression significantly attenuated the growth of PTEN-null PCa cells, including PC3, LNCaP, and C4-2 cells (Figure 2A). In contrast, BRG1 KD did not alter the growth of PTEN-WT PCa cells (22RV-1, BPH-1, and LAPC4 cells; Figure 2A). A similar dependency on BRG1 was confirmed in anchorage-independent growth assays. Depletion of BRG1 in PC3 and LNCaP cells, but not 22RV-1 cells, profoundly inhibited colony formation (Supplemental Figure 2B). Importantly, we showed that restoration of PTEN in PTEN-null cells (PC3 and LNCaP cells) rendered them insensitive to BRG1 downregulation (Supplemental Figure 2C). We next asked whether the protumorigenic functions of BRG1 are dependent on its chromatin-remodeling activity. Reexpression of WT, but not ATPase-deficient BRG1, restored the defects in colony formation and cellular migration of BRG1-depleted cells (Figure 2B).

Given the genetic and epigenetic heterogeneity of PCa cells, we ablated BRG1 alone or in combination with PTEN in the same cells. Consistently, PTEN KD accelerated cellular proliferation as predicted, and BRG1 loss alone did not have any discernible effects on the growth of 22RV-1 and LAPC4 cells (Figure 2C). In sharp contrast, PTEN KD greatly sensitized the cells to BRG1 depletion. We showed that PTEN/BRG1 double KD curtailed cell growth to basal levels (Figure 2C). This result was strengthened by the xenograft assay results. We showed that PTEN-KD 22RV-1 cells were vulnerable to BRG1 deletion, indicating a synthetic lethal relationship (Figure 2D). As reflected by tumor volume, ablation of BRG1 in PTEN-KD cells profoundly attenuated tumor progression. IHC analysis indicated that silencing BRG1 in PTEN-KD tumors led to a marked reduction in cell proliferation and an increase in apoptosis (as measured by Ki67 levels and cleaved caspase-3 levels, respectively; Supplemental Figure 2D). To provide further evidence that
BRG1 is important in PTEN-null PCa, we also performed an intracardiac injection assay to determine whether BRG1 downregulation suppressed the metastatic potential of PTEN-null PC3 cells. As reflected by bioluminescence imaging (BLI), mice injected with BRG1-KD cells showed greatly reduced skeletal colonization of tumor cells (Figure 2E). Compared with the control subjects, the BRG1-KD group showed a substantial decrease in osteolysis (Figure 2F). Along with reduced skeletal metastasis (E-cadherin ${ }^{+}$ cells), positive tartrate-resistant acid phosphatase (TRAP) staining was decreased after loss of BRG1 (Figure 2G). Taken together, these results highlight that BRG1 is required for the tumorigenesis of PTEN-deficient PCa cells.

Brg1 loss impairs tumor progression in Pten-null mouse model. To further corroborate the BRG1-PTEN relationship in vivo, we utilized GEMs to explore the genetic interaction between BRG1 and PTEN. Hence, Brg1 $1^{\mathrm{f} / \mathrm{fl}}$ mice (34) were crossed with Probasin-Cre $\left(\mathrm{PB}^{\mathrm{Cre} /+}\right)$ mice to delete $\mathrm{Brg} 1$ in the prostate epithelium (Brg1 $1^{\mathrm{PC}-/-}$ mice). Histological examination of the prostate from 6-monthold $\mathrm{Brg} 1^{\mathrm{PC}-/-}$ mice indicated that inactivation of Brg1 alone did not lead to pathological abnormalities in prostate lobes ( $n=5$; Supplemental Figure 3A). Next, we crossed $\mathrm{Brg}^{\mathrm{PC}-/-}$ mice with $\mathrm{Pten}^{\mathrm{fl} / \mathrm{fl}}$ mice (referred to as $\mathrm{Pten}^{\mathrm{PC}-/}$; $\mathrm{Brg} 1^{\mathrm{PC}-/-}$ mice; Figure $3 \mathrm{~A}$ and Supplemental Figure 3B). To better visualize tumor progression, the compound mice were also crossed with Rosa26-LSL luciferase reporter mice. Five-month-old Pten ${ }^{\mathrm{PC}-/} ; \mathrm{Brg}^{\mathrm{PC}-/-}$ mice exhibited a more than 2- to 3-fold reduction in bioluminescence intensity in the prostate relative to that in $\mathrm{Pten}^{\mathrm{PC}-/-}$ mice (Figure 3B), indicating that loss of BRG1 inhibited tumor progression in a Pten-null mouse model. Histopathological examination verified that inactivation of Brg1 impaired the progression of Pten-null prostate tumors. Regions of HGPIN, characterized by the intraglandular proliferation of crowding cells with nuclear atypia, developed in the prostate of 5-month-old $\mathrm{Pten}^{\mathrm{PC}-/-}$ animals (Figure 3, C and D). In addition, 3 out of $12 \mathrm{Pten}^{\mathrm{PC}-/-}$ mice developed invasive adenocarcinoma, as judged by a subset of tumor cells breaking the basal membrane (indicated by SMA $\alpha$ staining) and invading through the prostatic stromal (androgen receptor [AR] staining, arrow) (Figure 3E). In contrast, mice with inactivation of both Pten and Brg1 developed lesions largely arrested at the hyperplasia or lowgrade PIN (LGPIN) stage with incomplete penetrance (Figure 3, $\mathrm{C}$ and D). Likewise, the development of invasive adenocarcinoma was not evident in $\mathrm{Pten}^{\mathrm{PC}-/}$; $\mathrm{Brg} 1^{\mathrm{PC}-/-}$ mice (Figure 3E). Similarly, the decreased proliferative index was confirmed in the prostate of $\mathrm{Pten}^{\mathrm{PC}-/}$; $\mathrm{Brg}^{\mathrm{PC}-/-}$ mice relative to that in $\mathrm{Pten}^{\mathrm{PC}-/-}$ mice by Ki67 staining (Supplemental Figure 3C). Collectively, our results demonstrate that BRG1 is critical for the progression of prostate tumors initiated by PTEN deficiency.

To determine the cell-autonomous role of BRG1 in PTEN-deficient tumors, we utilized an organoid platform in which mini-prostates largely recapitulate the histological features of the prostate, including multilayered structures with nuclear AR expression and p63 expression in basal cells $(35,36)$. We isolated luminal cells from the prostate of TMPRSS2-Cre ${ }^{\text {ERT2-IRES-GFP }}$; Pten ${ }^{\mathrm{fl} / \mathrm{fl}}$ mice, as described previously $(35,36)$. Pten deletion was achieved by 4-hydroxytamoxifen (4-OHT), and Brg1 was knocked down via lentiviral transduction (Figure 3F and Supplemental Figure 3D). As predicted, Pten loss facilitated organoid formation, and BRG1 
A

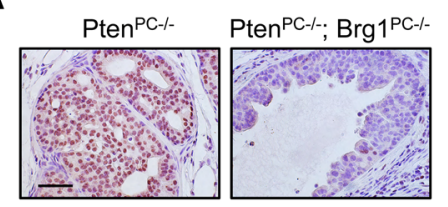

D

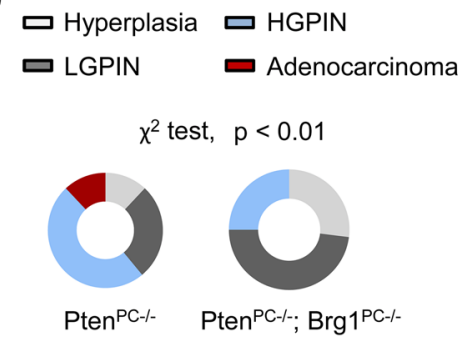

B

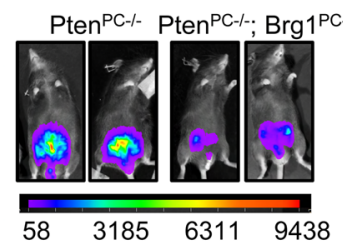

E

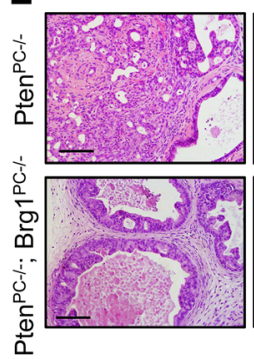

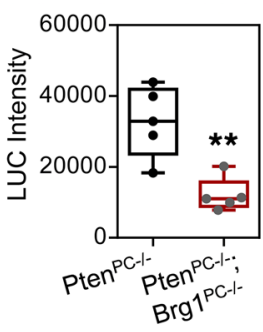
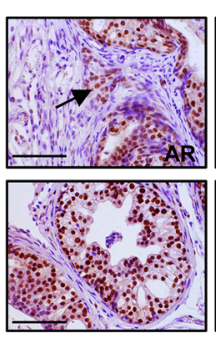
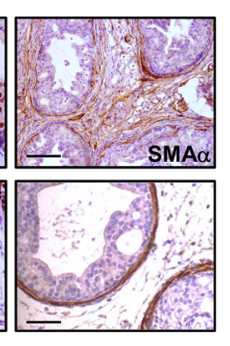

C

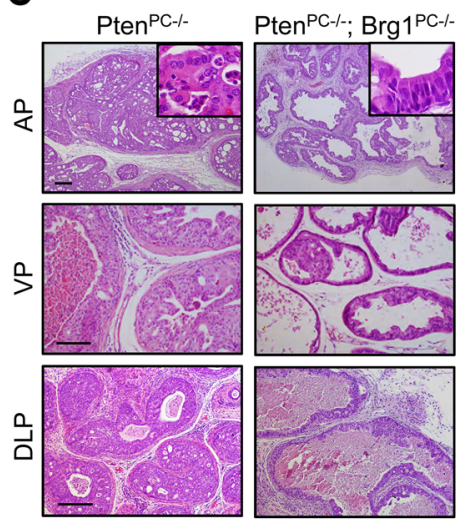

F

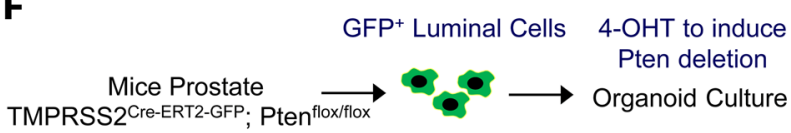

EV

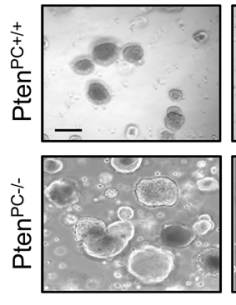
ShBRG1
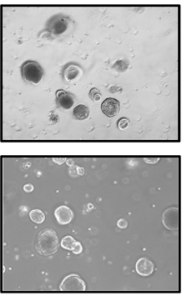

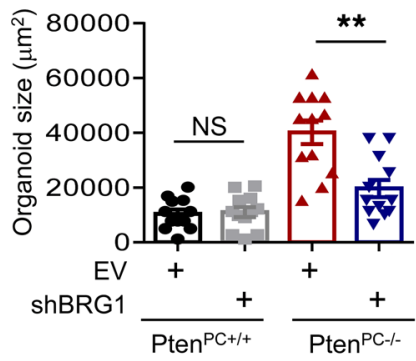

G
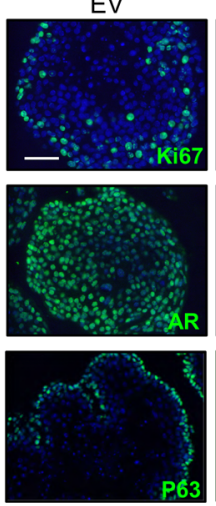

Pten ${ }^{\mathrm{PC}}$
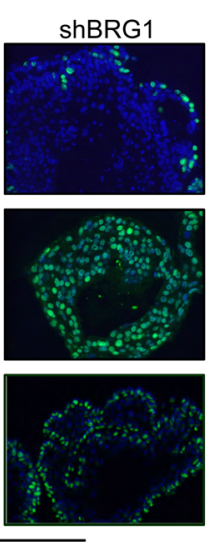

H
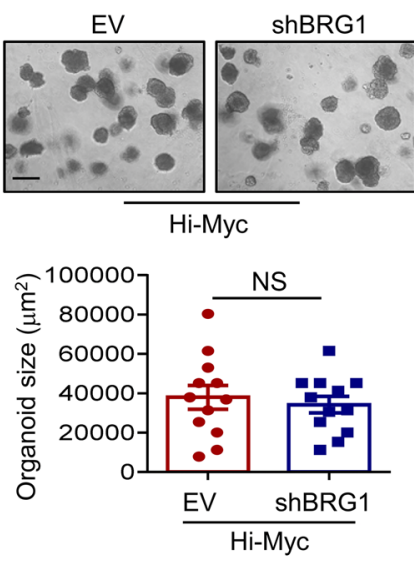

Figure 3. Brg1 loss inhibits Pten loss-induced tumorigenesis in mice. (A) IHC staining of BRG1 in prostate sections of 5-month-old Pten ${ }^{\mathrm{PC}-{ }^{-}}$, and Pten ${ }^{\mathrm{PC}--_{-}}$; $\mathrm{Brg}^{\mathrm{PC}-1-}$ mice. Scale bar: $50 \mu \mathrm{m}$. (B) Representative BLI images of Pten ${ }^{\mathrm{PC}-1-}$, and Pten ${ }^{\mathrm{PC}-1-}$; Brg ${ }^{\mathrm{PC}-1-}$ mice at 5 months of age. Quantification of $\mathrm{BLI}$ is shown on the right ( $n=5$, 2-tailed Student's $t$ test). (C) H\&E-stained sections of representative anterior prostate (AP), dorsal-lateral prostate (DLP), and ventral prostate (VP). Scale bars: $100 \mu \mathrm{m}$. Original magnification for the inset, $\times 50$. (D) Quantification of histology grade in Pten ${ }^{\mathrm{PC}-I_{-}}(n=10)$, and Pten $n^{\mathrm{PC}-l^{-}} ; \mathrm{Brg}^{\mathrm{PC}-l_{-}}$mice $(n=6)$ at 5 months of age $\left(\chi^{2}\right.$ test). (E) H\&E, AR, and SMA $\alpha$ staining of the DLP in Pten ${ }^{\mathrm{PC}-I_{-}}$(with the appearance of invasive adenocarcinoma) and Pten ${ }^{\mathrm{PC}-I^{-}}$; Brg1 ${ }^{\mathrm{PC}-I_{-}}$ mice. Scale bars: $100 \mu \mathrm{m}$. (F) Representative images of organoids from Pten-WT and Pten-null prostates with or without BRG1 KD (shBRG1). Quantitation of organoid size is representative of 3 experiments shown on the right (2-tailed Student's $t$ test). Scale bar: $200 \mu \mathrm{m}$. (C) Immunostaining of Ki67, AR, and P63 as indicated. Scale bar: $50 \mu \mathrm{m}$. (H) Organoid images derived from prostatic Myc-overexpressing mice (Hi-Myc) with or without BRG1 KD (shBRG1); quantitative results are representative of 3 experiments shown at the bottom (2-tailed Student's $t$ test). Scale bar: $400 \mu \mathrm{m} .{ }^{* *} P<0.01$.

depletion did not affect the initiating capacity or size of Pten-WT organoids (Figure 3F). In contrast, we found that BRG1 ablation profoundly reduced the clonogenic potential of Pten-deficient organoids, as reflected by organoid size and the number of $\mathrm{Ki}^{+}$ cells (Figure 3, F and G). To further determine whether Brg1 is specifically required for Pten-deficient tumors, we evaluated the consequence of BRG1 KD in organoids derived from prostatic c-Mycoverexpressing mice (37). Myc amplification or overexpression is commonly found in human tumors $(2,3)$. Thus, this model likely represents a subset of human PCa distinct from that driven by PTEN loss. Indeed, we showed that Brg1 ablation did not alter the tumorigenic potential elicited by c-Myc overexpression (Figure $3 \mathrm{H}$ and Supplemental Figure 3E). Next, we asked whether other common genetic changes in PCa, such as p53 deletion or SPOP mutation, could affect the synthetic relationship between PTEN and BRG1. To this end, we used the organoid model generated from
Pten and Tp53 double-KO mice (Pten ${ }^{\mathrm{PC}-/-}$; Tp53 ${ }^{\mathrm{PC}-/-}$ ) and found that BRG1 KD still inhibited the growth of organoid (Supplemental Figure 3F). Likewise, overexpression of patient-derived SPOP mutants (F133V) in PC3 and 22RV-1 PTEN-KD cells did not alter the sensitivity to BRG1 downregulation (Supplemental Figure 3G). Thus, GEMs and organoid assays collectively demonstrate the critical and specific functions of Brg1 in Pten-mutated PCa.

PTEN loss modulates the AKT-GSK3 $\beta$ signaling axis to increase $B R G 1$ protein stability. Next, we aimed to explore the molecular basis by which PTEN loss sensitizes cells to BRG1 ablation. We noticed that BRG1 protein levels were higher in PTEN-null PCa cells (PC3, LNCaP, and C4-2 cells) than in normal prostate epithelial cells (RWPE-1 cells and the mouse anterior prostate [mAP]) or PTEN-WT PCa cells (BPH-1, 22RV-1 and LAPC4 cells) (Figure 4A). Similar results were observed in the $\mathrm{Pten}^{\mathrm{PC}-/-}$ mouse prostate. The levels of BRG1 protein, not mRNA, were markedly 
A

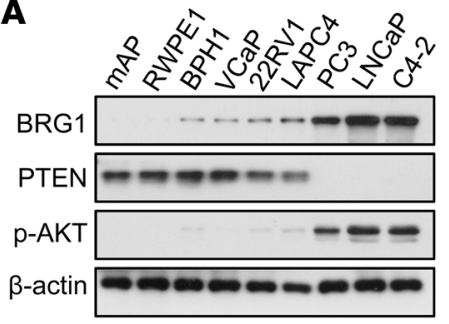

B

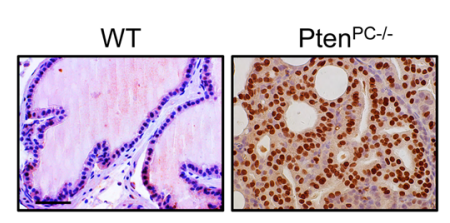

C

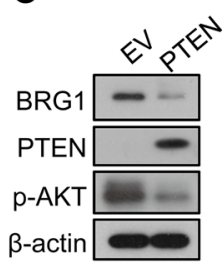

D

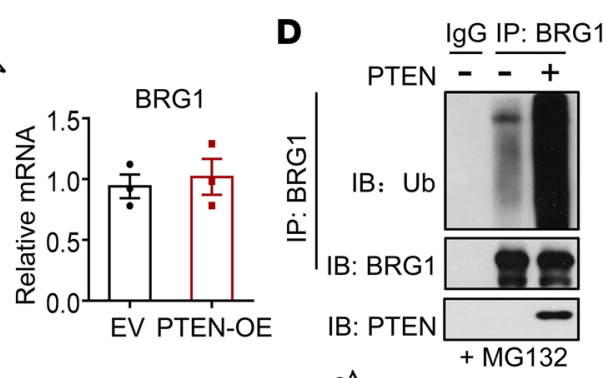

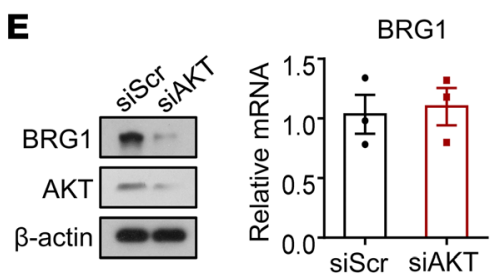

H

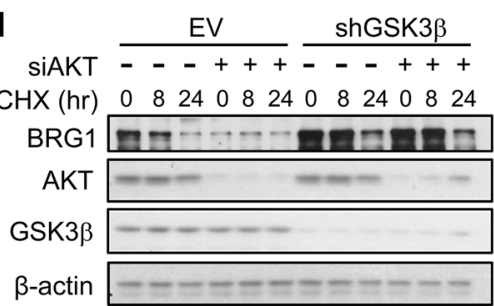

PC3

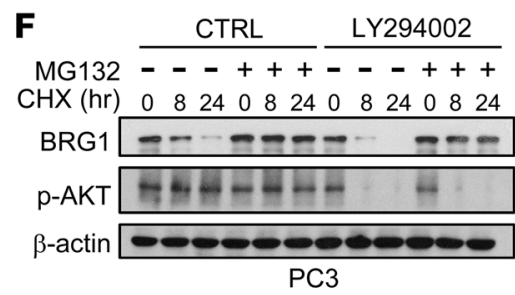

PC3
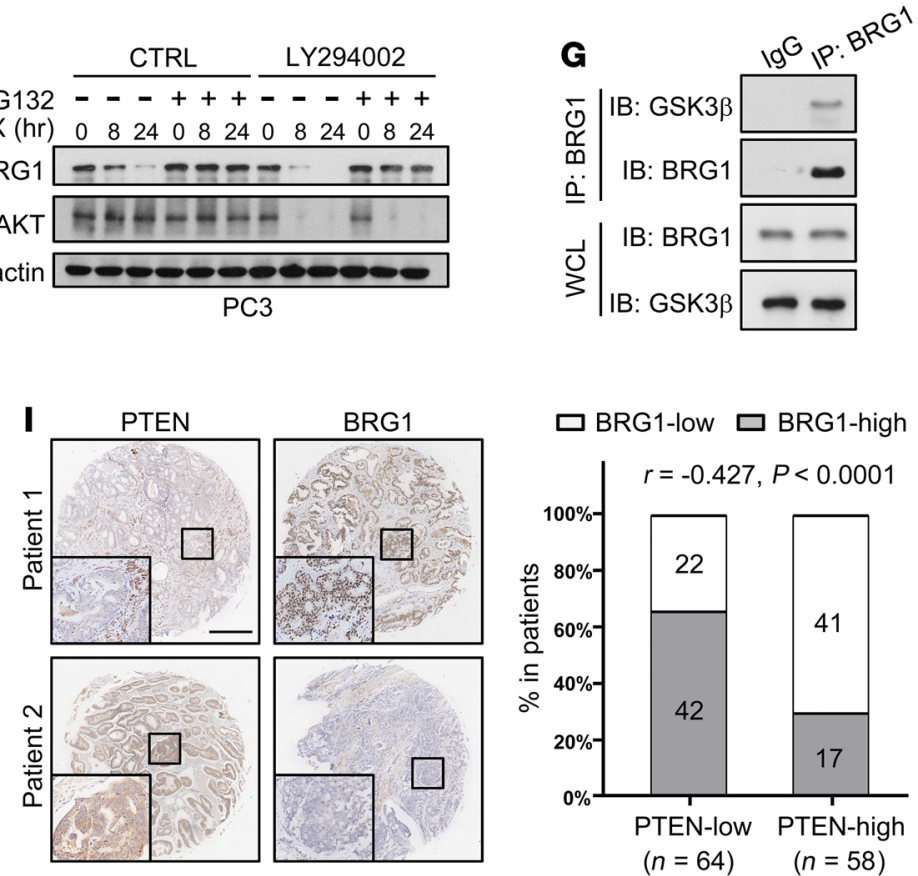

Figure 4. PTEN/AKT/GSK3 $\beta$ modulates BRG1 stability in PCa cells. (A) IB analysis of the indicated proteins in mouse AP lysates (mAP) and human prostate cell lines as indicated. (B) Representative BRG1 staining in prostate sections of WT and Pten ${ }^{\mathrm{PC}-1-}$ mice. Scale bar: $50 \mu \mathrm{m}$. (C) IB as indicated (left) and BRG1 mRNA levels (right) in control and PTEN-overexpressing PC3 cells. (D) Detection of BRG1 ubiquitination by IP and IB as indicated. (E) Examination of BRG1 protein and mRNA in PC3 cells transfected with scramble or AKT oligonucleotides. (F) IB analysis of PC3 cells treated with DMSO or $20 \mu \mathrm{M}$ LY294002 with or without MC132. (G) IB analysis of whole cell lysates (WCL) and IP from PC3 cells as indicated. (H) IB analysis of PC3 cells transfected with scramble or AKT oligonucleotides with or without GSK3 $\beta$ KD at the indicated times. (I) Representative images of BRG1 and PTEN staining. The box plot shows the relative BRG1 level in PTEN-low and PTEN-high patients (an Asian radical prostatectomy cohort). Kendall's tau-beta was used to test for correlations in the IHC results. Scale bar: $250 \mu \mathrm{m}$. Quantitative data from experiments performed in triplicate, 2-tailed Student's $t$ test (C and E).

increased in Pten-deficient prostates relative to WT controls (Figure 4B and Supplemental Figure 4A). Importantly, reintroduction of PTEN in PC3 cells led to a profound decrease in BRG1 protein, together with an increase in BRG1 polyubiquitination, without affecting BRG1 mRNA levels (Figure 4, C and D). These observations indicate that PTEN governs BRG1 expression by posttranscriptional regulation.

In the context of PTEN loss, the most pronounced signaling event is the constitutive activation of AKT, which influences proteasome-dependent protein degradation $(33,38)$. Indeed, we showed that the inhibition of AKT signaling by siRNA-KD or LY294002 treatment attenuated BRG1 stability without changing its mRNA level (Figure 4, E and F). Consistently, cotreatment of cells with MG132 and LY294002 prevented the degradation of BRG1 protein (Figure 4F), emphasizing that PTEN loss activates the AKT axis to stabilize BRG1 in a proteasome-dependent man-

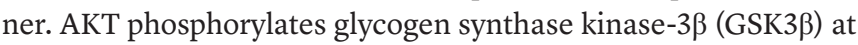
serine 9 and inhibits its kinase activity (39-41). We therefore spec- ulated that PTEN loss stabilizes BRG1 by impairing GSK3 $\beta$ activity. In line with this possibility, we first demonstrated that BRG1 interacted with GSK3 $\beta$ in cells (Figure 4G). GST pull-down analysis revealed that the carboxy-terminus of BRG1 (aa 1300-1647) was responsible for the direct association between BRG1 and GSK3 $\beta$ (Supplemental Figure 4B). Functionally, overexpression of WT or constitutively active GSK3 $\beta$ (GSK3B-S9A) attenuated the half-life of BRG1 protein in HEK293 cells (Supplemental Figure 4C). More importantly, depletion of GSK3 $\beta$ in AKT-KD PC3 cells prolonged the half-life of BRG1 protein, indicating that PTEN-AKT stabilized BRG1 via GSK3 $\beta$ (Figure 4H). The regulation of BRG1 by PTEN was reinforced by patient specimen analysis. A negative correlation between BRG1 and PTEN protein was observed in TMAs consisting of 122 PCa samples $(r=-0.427, P<0.0001$; Figure $4 \mathrm{I})$. Together, our results demonstrate that the PTEN/AKT/GSK3 $\beta$ signaling axis governs BRG1 stability in PCa cells. Thus, BRG1 is upregulated in PTEN-deficient tumors, which might cause the cells to rely on BRG1 presence. 
A

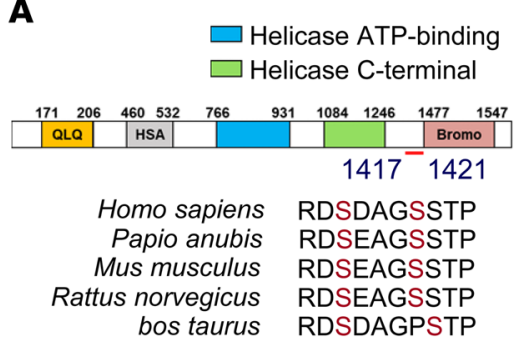

B

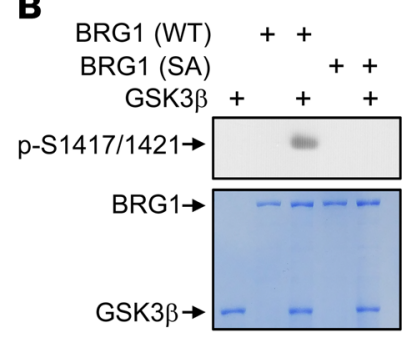

C
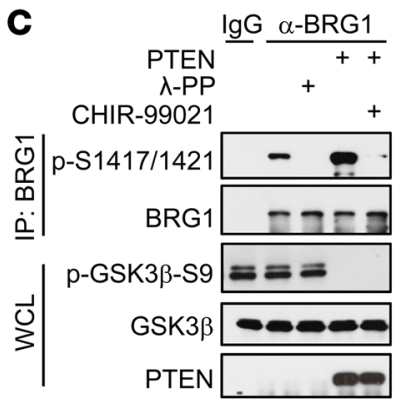

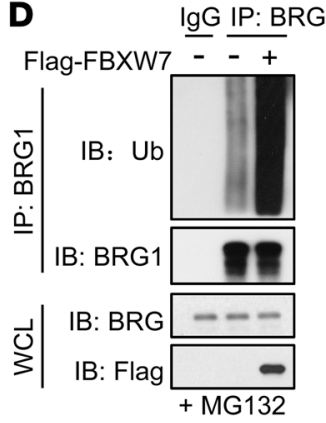

$\mathbf{E}$

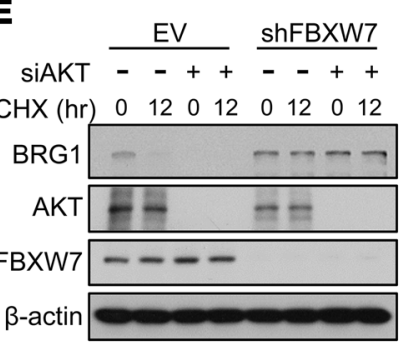

F $\quad B R G 1(W T)++$

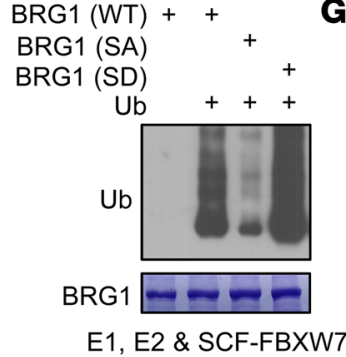

G Flag-BRG1 (WT) Flag-BRG1 (SA) Flag-BRG1 (SD)

$$
\text { HA-FBXW7 + }
$$

\begin{tabular}{l|l} 
至| IB: Flag \\
$\dot{\underline{Q}} \mid$
\end{tabular}

$\begin{array}{ll}\text { II } & \text { IB: HA } \\ & \text { IB: HA }\end{array}$ \begin{tabular}{c|c} 
IB: HA \\
$\vdots$ & IB: Flag
\end{tabular}

E1, E2 \& SCF-FBXW7

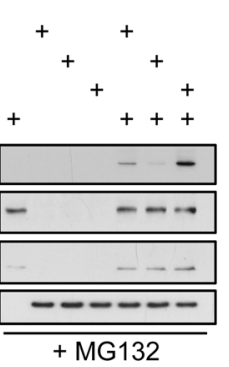

+ MG132
H

WT SA-1 SA-2 SD-1 SD-2 $\mathrm{CHX}(\mathrm{hr}) \overline{024} \stackrel{024}{024} \overline{024} \frac{024}{0 .}$ BRG1 short ex BRG1 long ex

$\beta$-actin

I

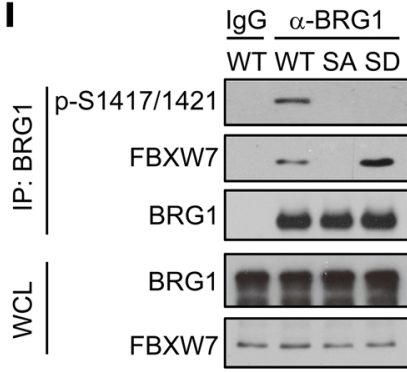

$J$

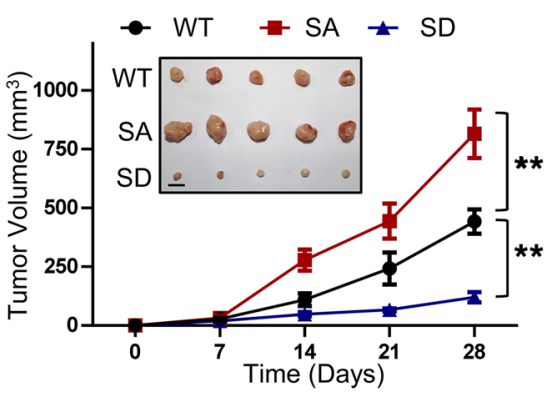

K

Patient 1223445667889101112

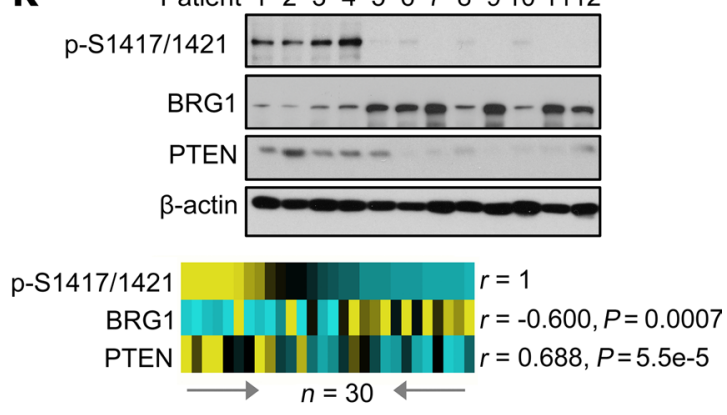

Figure 5. The PTEN/AKT/CSK3 $\beta$ axis modulates BRG1 stability through the FBXW7-dependent ubiquitin proteasome pathway. (A) Sequence alignment of the putative GSK3 $\beta$ phosphorylation sites at S1417 and S1421 of BRG1. (B) In vitro kinase assays depicting major GSK3 $\beta$ phosphorylation sites in BRG1. (C) IB analysis of WCL and immunoprecipitates from control and PTEN-overexpressing PC3 cells treated with CHIR-99021 or $\lambda$-phosphatase as indicated. (D) Lysates from control and FBXW7-overexpressing cells were subjected to IP with an anti-BRG1 antibody, and ubiquitinated BRG1 was detected by an anti-Ub antibody. (E) IB analysis of PC3 cells transfected with scramble or AKT oligonucleotides with or without FBXW7 KD (shFBXW7). (F) Flag-tagged WT, BRG1-SA, and BRG1SD proteins were incubated with SCF-FBXW7 complex as indicated and then subjected to Western blotting. (G) IB analysis of the indicated protein in WCL and immunoprecipitates from 293T cells transfected with HA-tagged FBXW7 and Flag-tagged BRG1, BRG-SA, or BRG1-SD. (H) IB analysis of the indicated protein in WT, SA, and SD cells. (I) WT, SA, and SD cell lysates were subjected to IP with the indicated antibodies. (J) Volume of subcutaneous tumors derived from WT, SA, and SD cells ( $n=6$, 2-way ANOVA followed by Tukey's multiple comparisons test). Scale bar: $1 \mathrm{~cm}$. (K) Representative image of BRG1, P-1417/1421, and PTEN expression in lysates from PCa samples (upper panel). Pearson's correlations among BRG1, P-1417/1421, and PTEN in PCa specimens are summarized in the heatmap $(n=30)$. ${ }^{* *} P<0.01$.

PTEN loss stabilizes BRG1 through inhibition of the GSK3B/ FBXW7-mediated ubiquitin proteasome pathway. We sought to determine the regulatory mechanism by which PTEN/AKT/ GSK3 $\beta$ modulates BRG1 stability. Previous studies have reported that GSK3 $\beta$ directly phosphorylates its substrates, which facilitates recognition by an $\mathrm{E} 3$ ligase for subsequent protein destruction (24, $41,42)$. Interestingly, consensus sequence alignment revealed an evolutionarily conserved GSK3 $\beta$ phosphorylation sequence (XpS/ TGXXpS/T) at S1417 and S1421 in the carboxy terminus of BRG1 (Figure 5A). Mass spectrometry experiments verified that both S1417 and S1421 were phosphorylated in HEK293 cells, and these phosphorylation signals were further enhanced upon GSK3 $\beta$ overexpression (Supplemental Figure 5A). To determine whether GSK3 $\beta$ indeed directly phosphorylates BRG1, we performed in vitro kinase assays. The results showed that only WT BRG1, not the mutant BRG1-S1417A/S1421A (BRG1-SA), was efficiently phosphorylated by GSK3 $\beta$ (Figure 5B), supporting a direct role for GSK3 $\beta$ in phosphorylating BRG1 at S1417/1421. We further generated an antibody that specifically recognized phosphorylation of BRG1 at S1417/1421 (Supplemental Figure 5B). Restoration of PTEN in PC3 cells profoundly enhanced endogenous phospho-BRG1-S1417/1421 levels, whereas inhibition of GSK3 $\beta$ kinase activity by CHIR-99021 treatment efficiently blunted this phosphorylation event (Figure 5C). Notably, $\lambda$-phosphatase treatment verified that the detected phosphorylation signal was specific (Figure $5 \mathrm{C}$ ).

FBXW7 and $\beta$-TrCP are known Skp1-Cullin1-F-box protein E3 ubiquitin ligases involved in PTEN/GSK3ß-mediated protein destruction $(24,43,44)$. Using an siRNA-KD approach, we found 
A

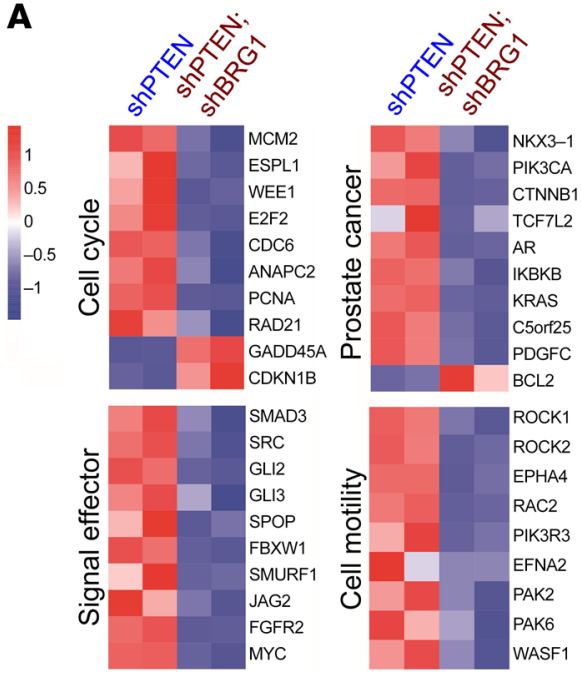

B

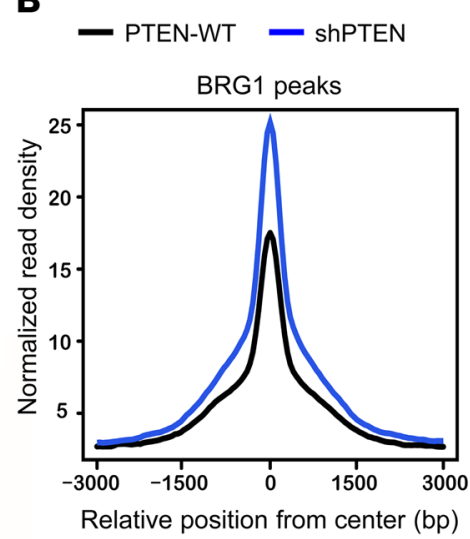

C

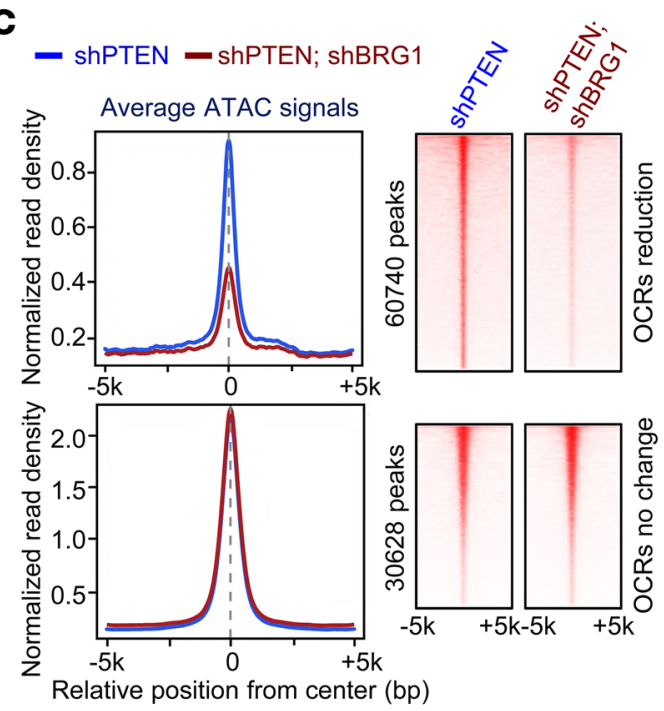

D

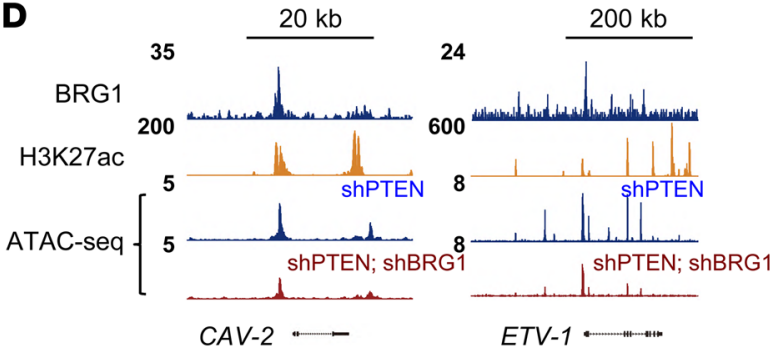

E

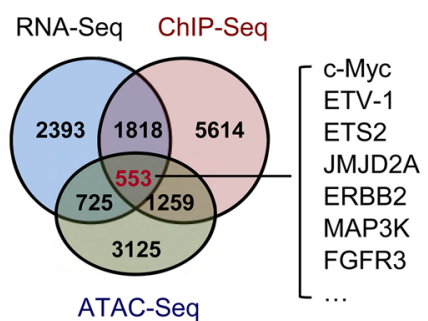

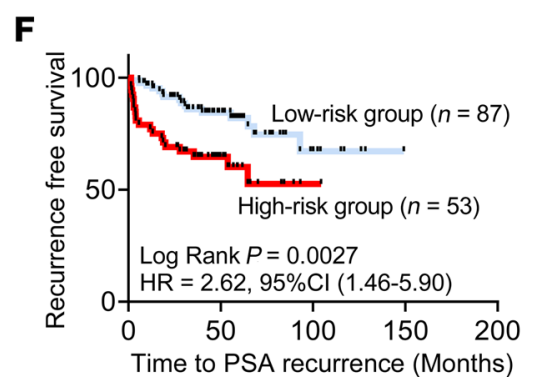

Figure 6. BRG1 remodels the chromatin configuration to influence a protumorigenic transcriptome in PTEN-deficient PCa cells. (A) Heatmap summarizing independent RT-qPCR results, as indicated. (B) ChIP-Seq summary plot of BRG1-binding intensities across BRG1 peaks in control and PTEN-KD (shPTEN) 22RV-1 cells. (C) ATAC-Seq signals showing the profiles of OCRs across the indicated genomic peaks in PTEN-KD and PTEN; BRG1-KD (shPTEN; shBRG1) cells. Heatmap summarizing ATAC-Seq signals displayed from $-5 \mathrm{~kb}$ to $+5 \mathrm{~kb}$ surrounding the center of each peak (right), which was sub-grouped based on the changes after BRG1 ablation. 60,740 Peaks (more than $60 \%$ of total) showed decreased DNA accessibility after BRG1 ablation (right top panel). (D) ChIP-Seq tracks of BRG1, H3K27ac, and ATAC-Seq signals in CAV-2 and ETV-1 gene loci, as indicated. (E) Venn diagram indicating overlapping genes with BRG1 peaks and changes in OCRs and DEGs. (F) PTEN-dependent BRG1 signature stratifies the patients (by K-mean clustering) for biochemical relapse (CSE21032; $P$ values by log-rank test).

that silencing FBXW7, not $\beta$-TrCP, greatly enhanced BRG1 protein levels in PCa cells (Supplemental Figure 5C). Similarly, FBXW7 overexpression stimulated the polyubiquitination of BRG1 in PC3 cells (Figure 5D). Consistently, ablation of FBXW7 restored BRG1 protein levels in AKT-depleted cells (Figure 5E), establishing a causal link between PTEN-AKT and FBXW7 in modulating BRG1 stability. Next, we conducted an in vitro ubiquitination assay to determine whether FBXW7 indeed targets BRG1 for degradation upon phosphorylation by GSK3$\beta$. IB with anti-ubiquitin antibodies revealed that polyubiquitinated forms of BRG1 were markedly reduced when alanine was substituted for serine (BRG1-S1417A/ S1421A, BRG1-SA), whereas the phospho-mimic BRG1-S1417D/ S1421D (BRG1-SD) protein exhibited significantly more polyubiquitination than the WT protein (Figure 5F). Similarly, exogenously expressed BRG1-SD protein displayed an enhanced turnover rate compared with that of WT BRG1 protein, whereas BRG1-SA was more stable in HEK293 cells (Supplemental Figure 5D). We further demonstrated that P-S1417/1421-BRG1 facilitated the direct binding between FBXW7 and BRG1 in HEK293 cells. BRG1-SA was dissociated from FBXW7, whereas the phospho-mimic BRG1SD protein exhibited enhanced binding to FBXW7 (Figure 5G).
To substantiate this observation, we substituted S1417/1421 in endogenous BRG1 for Ala or Asp in PC3 cells by CRISPR-Cas9 technology and thereby generated SA and SD cells, respectively (Supplemental Figure 5E). Indeed, BRG1-SD protein exhibited more pronounced degradation and polyubiquitination, whereas BRG1-SA was more stable than its WT counterpart (Figure 5H and Supplemental Figure 5F). MG132 treatment attenuated the accelerated degradation of BRG1-SD protein (Supplemental Figure 5G). Along with the diminished phosphorylation of BRG1 in SA cells, there was less binding to FBXW7, whereas the SD mutant showed increased binding to FBXW7 (Figure 5I). Together, these results indicate that the phosphorylation of BRG1-S1417/1421 mediated by GSK3 $\beta$ facilitates its recognition by the SCF-FBXW7 E3 ligase, leading to BRG1 destruction. The significance of BRG1-S1417/1421 phosphorylation was further evaluated in a xenograft model (Figure 5J). We showed that SA cells were more tumorigenic, whereas SD cell-derived xenografts were significantly smaller than those derived from WT PC3 cells. Further examination of PCa specimens revealed the tight association between PTEN and phospho-S1417/1421 levels in 30 PCa specimens $\left(r=0.688, P=5.5 \times 10^{-5}\right.$; Figure $\left.5 \mathrm{~K}\right)$. In addition, an inverse correlation was observed between BRG1 and phos- 

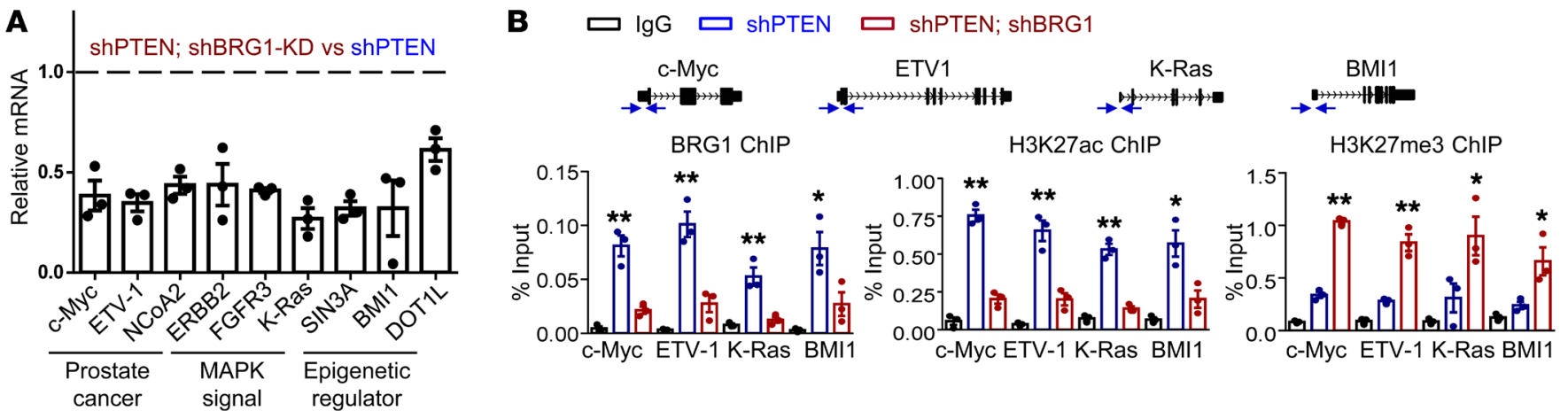

C

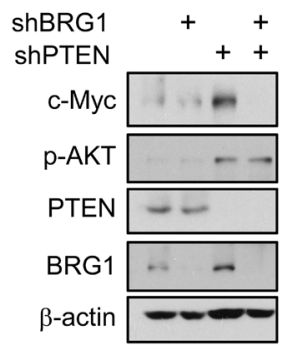

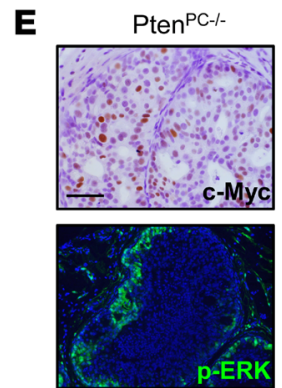

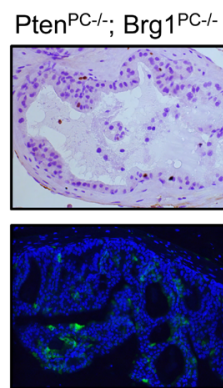

Figure 7. BRG1 modulates c-Myc and MAPK signaling in PTEN-deficient cells. (A) RT-qPCR analysis of gene expressions in PTEN-KD and PTEN; BRG1KD cells. (B) ChIP-qPCR assay of BRG1 binding, H3K27ac, and H3K27me3 in genes, as indicated. Arrows indicate primer locations. (C) IB analysis of the indicated protein in PTEN-KD and PTEN; BRG1-KD cells. (D) IB analysis of p-ERK levels in response to FGF treatment in control and PTEN-KD 22RV-1 cells with or without BRG1 KD (shBRG1). (E) IHC staining of c-Myc and p-ERK in prostate sections from Pten ${ }^{\mathrm{PC}-I^{-}}$, and Pten ${ }^{\mathrm{PC}-I_{-}}$; Brg1 ${ }^{\mathrm{PC}-I_{-}}$mice. Scale bar: $50 \mu \mathrm{m}$. Quantitative data from 3 independent experiments, 2-tailed Student's $t$ test (A and $\mathbf{B}$ ). ${ }^{*} P<0.05 ;{ }^{*} P<0.01$.

pho-S1417/1421 levels ( $\mathrm{r}=-0.600, P=0.0007$; Figure $5 \mathrm{~K})$. Cumulatively, these results establish that the PTEN/AKT/GSK3 $\beta$ axis regulates BRG1 degradation through the FBXW7-dependent ubiquitin proteasome pathway.

$B R G 1$ alters the transcriptome in PTEN-deficient cells to promote tumorigenesis. To investigate why PTEN-deficient tumors depend on BRG1 upregulation, we performed transcriptome profiling analysis in BRG1-depleted 22RV-1 cells with or without PTEN ablation. Through unsupervised cluster analysis of differentially expressed genes (DEGs), we found that the expression of 5489 genes was significantly altered in PTEN/BRG1 double-KD cells, whereas these genes showed distinct expression patterns in control, BRG1-KD, and PTEN-KD cells (Supplemental Figure 6A). In line with these findings, the KEGG-DEG relationship network indicated that BRG1 loss in PTEN-deficient cells significantly altered processes related to $\mathrm{PCa}$, cell cycle, and cell motility (Supplemental Figure 6B). The differential expression of selected genes was verified by quantitative reverse-transcriptase PCR (RT-qPCR), and the results were summarized in a heatmap (Figure 6A). To identify the downstream targets and pathways mediated by BRG1, we performed ChIP-Seq in control and PTEN-KD 22RV-1 cells. PTEN ablation led to a global increase in BRG1 occupancy at BRG1 peaks (Figure 6B and Supplemental Figure 6C). In total, 6279 genes (67.9\% BRG1-binding genes) showed enhanced BRG1 bindings in PTEN-KD cells than that in control cells. Given that BRG1 is a central ATPase subunit that mobilizes nucleosomes, we reasoned that BRG1 ablation in PTEN-deficient cells might impair chromatin configurations. To test this possibility, assay for transposase-accessible chromatin using sequencing
(ATAC-Seq) was performed to compare open chromatin regions (OCRs) between PTEN-KD and PTEN/BRG1-KD cells. Plots of genome-wide ATAC-Seq intensities revealed that BRG1 ablation in PTEN-KD cells led to a greater than $60 \%$ reduction in OCRs compared with BRG1-intact cells. There were 60,740 peaks showing decreased DNA accessibility in PTEN/BRG1-KD cells relative to that in PTEN-KD cells (Figure 6C). A few representative tracts showed that BRG1 loss led to decreased DNA accessibility at the ETV-1 and CAV-2 gene loci (Figure 6D). Together, our results indicate that BRG1 loss leads to marked changes in chromatin dynamics in PTEN-deficient tumors.

To identify the putative targets of BRG1 in PTEN-deficient $\mathrm{PCa}$, we aligned the BRG1 peaks with the changes in OCRs and DEGs; 553 genes overlapped and were termed the PTENdependent BRG1 signature (Figure 6E). KEGG pathway analysis revealed that these genes were related to signals regulating pluripotency of stem cells, axon guidance, transcriptional misregulation in cancer, and the MAPK signaling pathway (Supplemental Figure 6D). Next, we showed that the PTEN-dependent BRG1 signature stratified the patients by K-means clustering into 2 groups that exhibited significant differences in risk for biochemical recurrence (Figure 6F). In addition, by comparing gene sets differentially expressed in PTEN-WT and PTEN-null tumors, we found that PTEN-null tumors exhibited high activity of the BRG1 signature (Supplemental Figure 6E).

$B R G 1$ regulates $c-M y c$ and MAPK signaling in PTEN-deficient cells. Although it is difficult to attribute BRG1 function to a discrete target gene, we measured the expression of several genes known to be important for prostate tumorigenesis. Genes repre- 
A 22RV-1 (PTEN-WT)

$\rightarrow$ 22RV-1 (shPTEN)

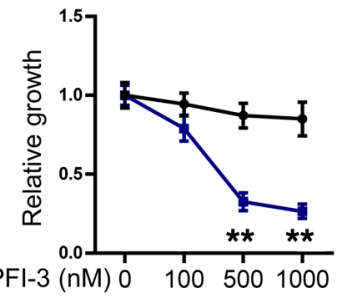

C

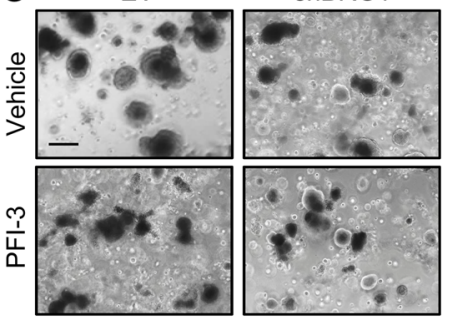

B $\quad \longrightarrow$ EV + Vehicle

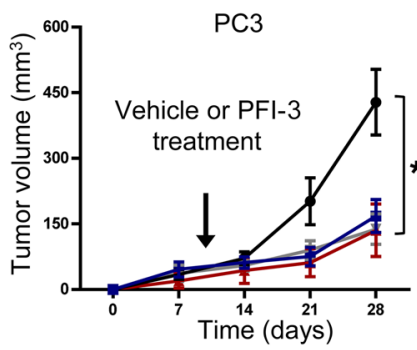

Pten $^{\mathrm{PC}-1-}$

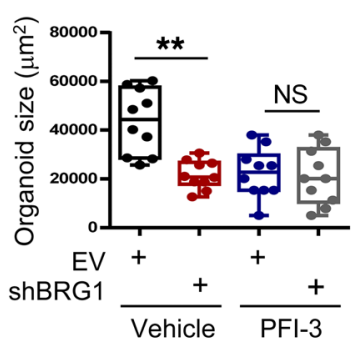

$-\mathrm{EV}+\mathrm{PFI}-3-$ shBRG1 + Vehicle

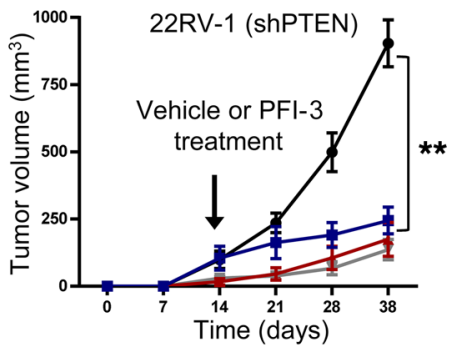

D

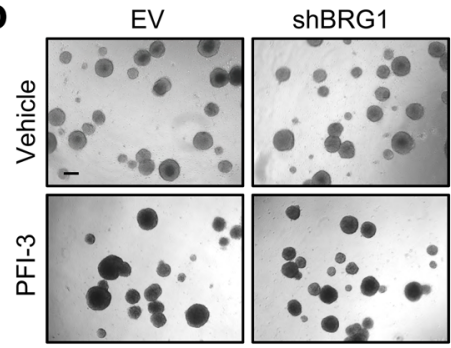

F Vehicle
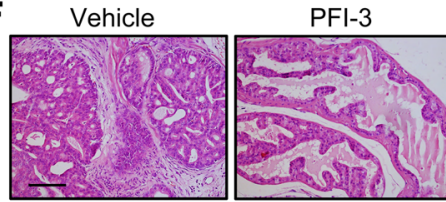

$\square$ Hyperplasia $\square$ HGPIN

$\square$ LGPIN $\square$ Adenocarcinoma $\chi^{2}$ test, $P<0.01$

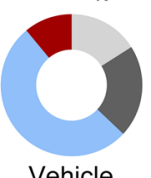

Vehicle

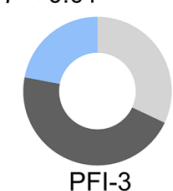

G

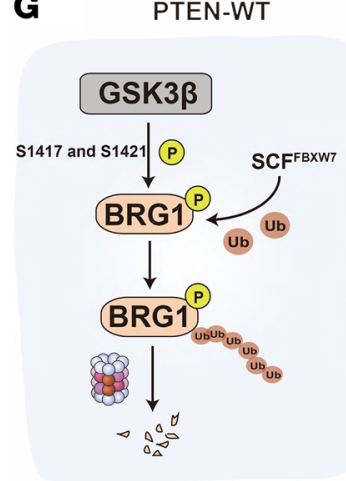

$-\operatorname{shBRG} 1+\mathrm{PFI}-3$

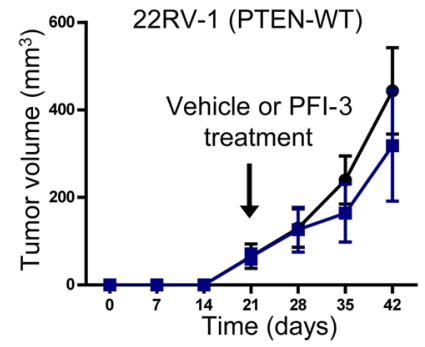

Hi-Myc

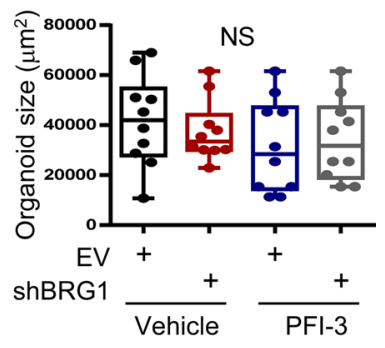

PTEN-deficiency

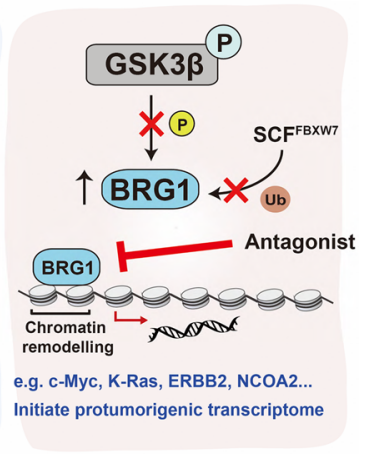

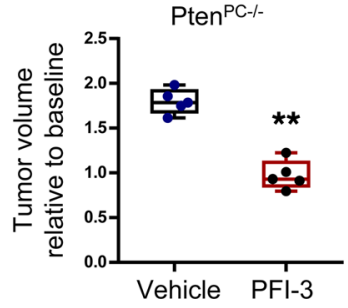

Figure 8. Targeting the SWI/SNF remodeling complex inhibits the progression of PTEN-deficient PCa. (A) Relative growth of PTEN-WT and PTENKD 22RV-1 cells treated with PFI-3 at different concentrations, as indicated. (B) Effects of PFI-3 treatment ( $50 \mathrm{mg} / \mathrm{kg}$, once per week) on xenografts, as indicated ( $n=6$ per group, 2-way ANOVA followed by Tukey's multiple comparisons test). Treatment started when tumors reached $50-100$ mm ${ }^{3}$. (C and $\mathbf{D}$ ) Representative images of PTEN-deficient (C) or Myc-overexpressing (D) organoids treated with vehicle or PFI-3 (100 nM; 7 days). Scale bar: $200 \mu$ m. (E) MRI analysis of prostates in Pten ${ }^{\mathrm{PC}-1-}$ mice treated with PFI-3 for 45 days $(50 \mathrm{mg} / \mathrm{kg}$, starting at 2.5 months of age; TO). Prostate tumors are indicated by red dotted circles, and relative tumor volume is shown at the bottom $(n=5,2$-tailed Student's $t$ test). Red asterisks indicate bladders. (F) H\&E staining of prostates from vehicle- and PFI-3-treated Pten ${ }^{\mathrm{PC}-1-}$ mice. Histology quantitation is indicated at the bottom $\left(n=5, \chi^{2}\right.$ test). Scale bar: $100 \mu \mathrm{m}$. (G) PTEN loss stabilized BRG1 through the inhibition of the AKT/GSK3 $\beta$ /FBXW7-mediated proteasome pathway. Consequently, BRG1 remodeled the chromatin configuration and initiated a PTEN-dependent BRG1 transcriptome to sustain tumor cell growth. Thus, targeting BRG1 represents a promising approach against PTEN-mutated prostate tumors. $n=3$ independent experiments, 2-tailed Student's $t$ test $(\mathbf{A}, \mathbf{C}$, and $\mathbf{D})$. ${ }^{*} P<0.05 ;{ }^{* *} P<0.01$.

sentative of key regulator in PCa, MAPK signal, and epigenetic regulator were chosen, including c-Myc, ETV-1, NCoA2, ERRB2, FGFR3, K-Ras, Sin3A, BMI1, and DOT1L (Figure 7A). Independent ChIP-qPCR experiments confirmed that BRG1 was present at these gene loci, consistent with a direct role for this subunit in SWI/SNF targeting (Figure 7B). ChIP-qPCR showed that BRG1 loss markedly reduced H3K9ac levels at the c-Myc, ETV-1, K-Ras, and BMI1 gene loci (Figure 7B). Because H3K27ac distinguishes active from poised and inactive chromatin (45), we consistently detected simultaneous increases in H3K27me3 at the same loci (Figure 7B). These results indicate that BRG1 loss favors a suppressive chromatin state to suppress gene expression. Focusing on the 
c-Myc and MAPK signals, we verified that c-Myc expression and phospho-ERK stimulation were downregulated to basal levels in PTEN/BRG1-KD cells compared with PTEN-deleted cells (Figure 7, C and D). Next, we extended our analysis to GEMs and showed that c-Myc and phospho-ERK levels were reduced in the prostates of $\mathrm{Pten}^{\mathrm{PC}-/-}$; $\mathrm{Brg}^{\mathrm{PC}-/-}$ mice relative to those in $\mathrm{Pten}^{\mathrm{PC}-/-}$ mice (Figure 7E and Supplemental Figure 6F). Moreover, a tight correlation between BRG1 transcriptome and genes linked to c-Myc and MAPK signaling was observed in PCa patients (Supplemental Figure 6G). Together, these data suggest that BRG1 remodels the chromatin configuration in PTEN-deficient PCa cells and initiates a protumorigenic transcriptome profile involving c-Myc and MAPK signaling, causing the cells to become dependent on BRG1.

BRG1 antagonist inhibits PTEN-deficient tumor progression. We investigated whether BRG1 inhibition with PFI-3, a cell-permeable inhibitor of BRG1 (SMARCA4) and BRM (also known as SMARCA2), has therapeutic efficacy in PTEN-deficient preclinical models. We found that depletion of PTEN greatly sensitized cells to PFI-3 treatment (Figure 8A). PFI-3 administration profoundly compromised the growth of PTEN-null PC3 cells and PTEN-depleted 22RV-1 cells, whereas no further inhibitory effects were seen in BRG1-depleted cells (Supplemental Figure 7A). Importantly, PFI3 treatment at the same concentration did not alter the growth of PTEN-WT 22RV-1 cells (Supplemental Figure 7A). This result was not surprising, as there is little or no druggable target (BRG1 protein) in WT 22RV-1 cells. Similar results were obtained in xenograft assays. Once the tumor size reached $50-100 \mathrm{~mm}^{3}$, mice received PFI-3 once a week and tumor volume was measured at the indicated time points (Figure 8B). PFI-3 administration to mice compromised the progression of xenografts derived from PTEN-deficient cells (PC3 and PTEN-KD 22RV-1 cells), whereas no effect was seen in xenografts derived from WT 22RV-1 cells (Figure 8B). Notably, we found that the antagonist did not cause further inhibitory effects in tumors derived from BRG1-depleted cells (Figure 8B). Next, using organoid assays, we showed that treatment with PFI-3 impaired the clonogenic potential of organoids derived from Ptennull mice, whereas c-Myc-overexpressing organoids showed no discernible response to PFI-3 (Figure 8, C and D). Similarly, PFI-3 treatment did not further reduce the size of BRG1-deficient organoids (Pten deletion or c-Myc overexpression context, Figure 8, C and D), indicating the specificity of PFI-3 for BRG1.

Finally, we carried out preclinical studies using $\mathrm{Pten}^{\mathrm{PC}-/-}$ mice, which were randomized to receive vehicle or PFI-3 at 2.5 months old, a time point when PIN is discernible. MRI indicated that PFI3 treatment led to a dramatic reduction in tumor volume (mean tumor volume reduction of approximately $43 \%$ ), with near-complete pathologic responses in all mice tested ( $n=5$; Figure $8 \mathrm{E})$. In contrast to the characterization of neoplasms in the vehicle group, neoplasms in PFI-3-treated mice were characterized as hyperplasia or LGPIN (Figure 8F). Consistently, antagonist-treated $\mathrm{Pten}^{\mathrm{PC}-/-}$ tumors displayed a drastic decrease in the frequency of Ki-67-positive mitotic cells along with an induction of apoptosis (Supplemental Figure 7B). Notably, PFI-3 treatment was tolerated well in mice because it did not cause significant weight loss, lethargy, or feeding abnormalities (Supplemental Figure 7, C and D). Thus, pharmaceutical inhibition of the SWI/SNF remodeling complex has beneficial results in a murine model of PCa.

\section{Discussion}

Cancer cells acquire molecular changes distinct from those in normal cells, which potentially exposes them to new epigenetic vulnerabilities (46). Thus, targeting chromatin regulators based on oncogene addiction can potentially lead to synthetic lethality in tumor cells $(21,47)$. It is well appreciated that cell identities are determined by transcription factors and epigenetic factors $(24,33)$, but how signaling molecules such as PTEN alter the cellular state is less understood. Our previous work demonstrated that PTEN loss can activate WHSC1, an H3K36 histone dimethyltransferase controlling the transcription of a large portion of genes (33). Here, we determined that BRG1, another chromatin modifier that controls transcription, is dysregulated in PTEN-deficient PCa cells (Figure 8G). Work from other labs has also identified CHD1 and EZH2 as PTEN targets (24, 48). Thus, it is intriguing to speculate that PTEN alters the cellular state by regulating epigenetic factors. We anticipate that more epigenetic factors will be identified under this paradigm. It is unlikely that PTEN loss by itself is sufficient to change cell identity; multiple gene mutations are usually required for full-blown tumorigenesis. It will be of great interest to investigate how other signaling molecules converge at transcriptional and epigenetic regulation to alter the cellular state. Ultimately, this knowledge will lead to the identification of novel therapeutic targets to selectively target tumor cells.

The role of BRG1 in cancer appears to be dependent on tumor type and subunit $(25,31)$. Frequent mutation and/or loss of expression of the catalytic subunit of BRG1 has been reported $(26,27)$. Nevertheless, an increasing number of studies have revealed the positive role of BRG1 in tumor initiation and progression (29, 30). Mammalian SWI/SNF enzymes regulate some constitutively expressed genes and are also closely linked to the reprogramming of gene expression in response to environmental or oncogenic insults (28). Using The Cancer Genome Atlas (TCGA) and other data sets, we found no statistical evidence for a mutually exclusive deletion pattern between PTEN and BRG1, possibly due to the low mutation or deletion frequency of BRG1 in PCa patients (Supplemental Figure 8A). Thus, BRG1 expression or its activity is potentially more important. Here, we showed that PTEN loss stimulates BRG1 expression through inhibition of the GSK3 $\beta$ /FBXW7-mediated ubiquitin proteasome pathway. Phosphorylation of BRG1 at S1417/1421 serves as a molecular switch to determine BRG1 protein levels in PCa cells. Thus, our results support the notion that synthetic lethal partners can also serve as downstream effectors of a tumor suppressor gene (24).

Our findings indicate that PTEN-deficient PCa cells are epigenetically distinct from PTEN-WT cells, with the former relying on BRG1 to modulate chromatin dynamics and gene expression. It is difficult to pinpoint the unique gene, if one exists, as the target of BRG1 responsible for tumorigenesis. Instead, our results suggest that multiple important oncogenes are regulated by BRG1. The c-Myc is a well-known positive regulator of cell growth, and its overexpression is commonly found in a wide variety of human cancers (49). In addition, ETS-2, NCoA2, and BMI1 are known to be important for prostate tumorigenesis $(4,50,51)$. In addition, studies have demonstrated that MAPK signaling is critical for prostate malignancy (13). We showed that several key molecules involved in MAPK signaling, including FGFR3, ERBB2, and KRAS, were deregulated in the absence of BRG1 and that phospho-ERK 
levels were decreased in the prostate of $\mathrm{Pten}^{\mathrm{PC}-/-}$; $\mathrm{Brg} 1^{\mathrm{PC}-/-}$ mice. Thus, as these genes are direct targets of BRG1 in PTEN-deficient PCa cells, it is not surprising that prostate tumorigenesis was largely compromised after BRG1 ablation.

Chromatin remodeling is instrumental in oncogenesis and can be therapeutically targeted. So far, specific pharmacologic inhibitors of BRG1 have not been developed. Given that PFI-3 is a potent antagonist of both BRG1 and BRM, we included BRG1depleted cells to address BRG1 dependence in the present studies. However, as tumor malignancy was already curtailed to basal levels after BRG1 loss, we cannot fully exclude BRM dependence in this setting. Nevertheless, we used an siRNA KD approach to deplete BRM in PCa cells and found that BRM depletion did not affect PCa cell growth (Supplemental Figure 8B). Therefore, PFI-3 is unlikely to have exerted its function on BRM. This result is not surprising, as BRG1 and BRM have distinct roles in many physiological and pathological processes. Inactivation of BRG1 is embryonic lethal, whereas inactivation of BRM results in viable animals without any overt deficiencies $(52,53)$. In addition, we also extended our analysis of PTEN and BRG1 in other tumor types. We found that BRG1 silencing inhibited the growth of PTEN-null glioma cells (U87MG and A172 cells), but not PTENWT glioma cells (LN-229 and LN-18 cells) (Supplemental Figure $8 \mathrm{C}$ ). In addition, PTEN KD greatly sensitized the melanoma (A375) and breast cancer cells (MCF7) to BRG1 inhibition (Supplemental Figure 8D). In summary, we establish a synthetical relationship between PTEN and BRG1 and provide a rational to develop BRG1 antagonists to treat PTEN-deficient PCa. Whether this observation holds true in other cancer types in vivo is of great interest for future investigation, considering that PTEN function is frequently disabled in a variety of tumors.

\section{Methods}

Please see the Supplemental Methods for information on expression plasmids, sgRNAs, siRNAs, ChIP assays, GST pull-down assays, IP, Western blotting, in vitro ubiquitination and kinase assays, primers (Supplemental Table 4), and mass spectrometry procedures.

Cell culture and treatments. Cells were all purchased from ATCC or Cell Bank, Shanghai Institutes for Biological Sciences, Chinese Academy of Sciences. Cells were cultured in standard DMEM or RPMI 1640 supplemented with $10 \%$ FBS. Scrambled, nontargeting siRNA was used as negative control for transient siRNA knockdown. Lentivirus was used to establish individual stable cells, and corresponding empty vectors were used as the controls for stable cell knockdown (by shRNA) or overexpression. Standard 24-well Boyden invasion chambers (BD Biosciences) were used to assess cell-migration abilities. For soft-agar colony-formation assay, cells were suspended in RPMI 1640 containing $0.35 \%$ low-melting agar (Invitrogen) and 10\% FBS and seeded onto a coating of $0.7 \%$ low-melting agar in RPMI 1640 containing 10\% FBS. Results were calculated based on 3 independent experiments. For MG132, CHX, and CHIR-99021 treatments, see descriptions in figure legends.

CRISPR screen. For the CRISPR screen, $4 \times 10^{6}$ PTEN-WT and PTEN-KD 22RV-1 cells were individually infected with the pooled lentiviral chromatin regulators library at a multiplicity of infection of 0.5. The library consists of 517 genes encoding epigenetic writers, erasers, and readers (at least 4 sgRNAs per gene; Supplemental Table 1) pur- chased from GV20 Oncotherapy Biotechnology Co. After 3 days of puromycin selection, the surviving cells were stored as 0-day control samples, and more than $3 \times 10^{6}$ cells (at least $\times 1000$ coverage) were passaged every 4 to 5 days (around 10 passages) during the continuous 45-day course. Then PCR was performed based on adaptor sequence to construct the sequencing library. Each library was sequenced at about 40 million reads to achieve approximately $\times 200$ average coverage over the CRISPR library, and data were analyzed by MAGeCK and MAGeCK-VISPR $(54,55)$. sgRNA counts were determined based on perfectly matched sequencing reads only. The sgRNA fold change was calculated by $\beta$ score ( $\log _{2}$ fold change $\geq 0.585$ ), and significance was defined as $P<0.01$.

Analysis of human TMAs. An Asian radical prostatectomy cohort was described previously $(32,33)$. Briefly, tissue samples and clinical parameters of $122 \mathrm{PCa}$ patients who underwent a radical prostatectomy were collected; these are listed in Supplemental Table 2. IHC analyses were performed using anti-BRG1 (Abcam, catalog ab110641) and anti-PTEN (Cell Signaling Technology, catalog 9559) antibodies. BRG1 and PTEN protein expressions were scored and quantified by pathologists blinded to the outcome of the cases. BRG1 protein quantification is based on a multiplicative index of the average staining intensity ( 0 to 3 ) and the extent of staining ( 0 to 3 ) in cores, yielding a 10-point staining index ranging from 0 (no staining) to 9 (strong staining). The staining score $\leq 4$ was defined as BRG1 low expression, whereas the score higher than 4 was referred as BRG1 high expression. PTEN protein was visually scored using a dichotomous scoring system as previously described (56). In brief, each spot of tumor tissue was scored as negative or positive for PTEN protein by comparing staining in malignant glands with that of adjacent benign glands and/or stroma, which provided an internal positive control within each tissue core. Staining was classified as negative if the intensity was markedly decreased or entirely negative across all tumor cells compared with the surrounding benign glands and/or stroma.

Animal experiments. All mice were maintained in a specific pathogen-free facility. For the subcutaneous xenograft model, PC3 cells $\left(5 \times 10^{5}\right)$ or $22 \mathrm{RV}-1$ cells $\left(1 \times 10^{6}\right)$ in a PBS/Matrigel mixture were injected into the flanks of male nude mice. Tumor size was measured every week, and the tumor volume was determined with the formula $0.52 \times L \times W^{2}$, where $L$ indicates length and $W$ indicates width. Nude mice aged 6 to 8 weeks received an intracardiac injection of $1 \times 10^{6}$ PC3 cells. BLI was performed with a NightOWL II LB 983 Imaging System (Berthold). Bone damage was detected by $\mathrm{x}$-ray radiography with a Faxitron instrument and quantified by Image J (NIH). Brg1 floxed mice (34) (provide by Pierre Chambon, Institut de Génétique et de Biologie Moléculaire et Cellulaire, Strasbourg, France) and

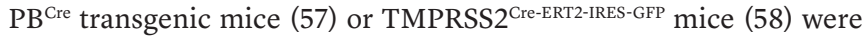
on the C57BL/6 background. Histology was graded using previously described nomenclature and criteria (59). In brief, HGPIN was characterized by intraglandular proliferation of crowding cells with atypia and cribriform formation or the development of multilayered solid glandular structures. Adenocarcinoma was indicated by the atypical cells that break the basal membrane. The quantitative results of histology were derived from 5 random slides for each mouse of the entire animal cohort, as described (60). Briefly, for each animal, 5 random fields were captured; these were further divided into 4 quadrants. In each quadrant, the most advanced histological feature (hyperplasia, LGPIN, HGPIN, or Adeno) was recorded for quantifi- 
cation. Thus, the numbers of each subtype of lesions in each experimental group were established, and the percentages of different subtypes of lesions were compared. Statistical significance between groups was determined by the $\chi^{2}$ test.

Mouse prostatic organoid generation and experiments. Prostate tissues were extracted from TMPRSS2 $2^{\text {Cre-ERT2-IRES-GFP }}$; Pten ${ }^{\mathrm{fl} / \mathrm{fl}}$ and TMPRSS2 $2^{\text {Cre-ERT2-IRES-GFP }} ;$ Pten $^{\mathrm{f} / / \mathrm{fl}} ; \mathrm{Tp} 53^{\mathrm{fl} / \mathrm{fl}}$ mice, and the digested cells were sorted by GFP to isolate luminal cells. The Myc-overexpressing organoids were generated from Hi-Myc mice as previously described (37). Cells were plated in Matrigel (BD) and covered with mouse media containing $50 \times$ diluted B27, $1.25 \mathrm{mM} \mathrm{N}$-acetyl-L-cysteine, $50 \mathrm{ng} / \mathrm{ml}$ EGF, 200 nM A83-01, 100 ng/ml Noggin, 500 ng/ml R-spondin 1, 10 $\mu \mathrm{M}$ Y-27632, and $1 \mathrm{nM}$ dihydrotestosterone. To delete Pten, CreERT2 was activated by adding $1 \mu \mathrm{M} 4-\mathrm{OHT}$ (Sigma-Aldrich) into the media overnight. Then the cells were infected with empty vector or shBRG1 lentivirus, and puromycin was used for selection. For organoid formation assays, 2000 cells were plated per well on day 1 , and the number and size of the organoids were determined on day 9.

RNA-Seq, ChIP-Seq, ATAC-Seq, and data analysis. Total RNA from 22RV-1 (PTEN-WT or PTEN-KD) cells with or without BRG1 ablation was subjected to HiSeq RNA-Seq, which was performed by BGI Tech Solutions Co. Each sample contained pooled RNA from 3 biological replicas and was mixed with an equal mass of RNA to minimize variation across samples. Transcriptome reads from RNA-Seq experiments were mapped to the reference genome (hg19) using the Bowtie tool. The gene expression level was quantified by the RSEM software package. We detected DEGs with the possion distribution method (61), which has been used in previous studies (62-64). Briefly, given that every gene's expression occupies only a small part of the library, the number of reads from gene A yields to the Poisson distribution. The probability of gene A expressed equally between 2 samples was calculated, after which the $P$ value could be corrected by Bonferroni's method. Meanwhile, correction for false positive and false negative errors was performed using the FDR method. We used FDR of 0.001 or less and the absolute value of the $\log _{2}$ ratio 1 or more as the default threshold to determine the significance of the gene expression difference. For the ChIP-Seq assay, chromatin was prepared from 3 biological replicates of control and PTEN-deleted 22RV-1 cells, and ChIP-Seq assays were then performed by Active Motif Inc. using an antibody against BRG1 (Abcam, catalog ab110641). Seventy-five nucleotide reads generated by Illumina sequencing were mapped to the genome using the BWA algorithm with default settings. RNASeq, ChIP-Seq, and ATAC-Seq data were deposited in the NCBI's Gene Expression Omnibus database (GEO GSE115619). The average profile for tag distributions was generated using SitePro and deeptools2 $(65,66)$. ATAC-Seq of PTEN-KD and PTEN/BRG1-KD 22RV-1 cells was performed by Romics. Quality filtered reads were then mapped to the GRCh37/hg19 reference genome using BWA (0.7.10). MACS2 was used to call peaks, and an initial threshold $q$ value of 0.01 was set as the cutoff.

Analysis of BRG1, MAPK, and c-Myc signatures in PCa patients. Analysis of human PCa data sets (Supplemental Table 3) was carried out essentially as previously described $(50,60)$. The BRG1 signature was derived from the gene expression profile of our own data set with a criteria fold change of 2 or more and an FDR of less than 0.001 . Genes upregulated or downregulated after U0126 treatment in NIH-
3T3 cells were defined as the MAPK signature (GEO GSE4739, fold change $\geq 1.5, P<0.05)$. The $\mathrm{c}-\mathrm{Myc}$ gene signature was derived from genes differentially expressed between $\mathrm{c}-\mathrm{Myc}$-transgenic and control mice (GEO GSE10954, fold change $\geq 1.5, P<0.05$ ). To define the degree of gene signature manifestation within the profiles from an external human tumor data set (e.g., TCGA), we used the previously described $t$ score metric $(50,60)$. For example, the $t$ score was defined for each external profile as the 2-sided $t$ statistic comparing the average of the BRG1-induced genes to the average of the BRG1-repressed genes (genes within the human tumor data set were first centered to standard deviations from the median of the primary tumor specimens). For a given data set, the $t$ score contrasted the patterns of the BRG1-induced genes against those of the BRG1-repressed genes to derive a single value denoting the coordinate expression of the 2 gene sets. The K-means clustering algorithm described previously (67) was used with the PTEN-dependent BRG1 signature to identify 2 cancer sample clusters.

PFI-3 treatment. Organoids were treated with PFI-3 (100 nM) after 2 days of culture, and the effects were measured after 7 days of treatment. For xenograft models, when the tumor volume reached $50-100 \mathrm{~mm}^{3}$, mice were randomized and treated with vehicle or PFI-3 (50 mg/kg body weight, 6-7 mice per group) by oral gavage every week. Tumor volume and weight were measured as mentioned above. For GEMs, Pten $^{\mathrm{PC}-/-}$ mice were administered vehicle or PFI-3 (50 mg/kg body weight) by oral gavage every Monday for a total of 45 days. MRI-based tumor volumes were reported for each mouse at time point zero (TO, study initiation) and time point 45 days (T45, study completion).

Statistics. All experiments were performed using 5-10 mice or 3 independently repeated experiments with cells. Unless otherwise indicated, the data in the figures are presented as the mean \pm SEM, and the results were analyzed by 2-tailed Student's $t$ test. Cox proportional hazards regression model analyses were performed with SPSS 22.0 statistical software. Statistical significance indicated in individual figures was determined by Student's $t$ test, 1-way ANOVA, 2-way ANOVA, Wilcoxon's signed-rank test, Pearson's correlation coefficient, log-rank test, or $\chi^{2}$ test. For all statistical tests, $P$ values of less than 0.05 indicate statistical significance.

Study approval. The use of pathological specimens and the review of all pertinent patient records were approved by the Institutional Ethics Review Board of Renji Hospital, and informed consent was obtained from the patients. All animal experiments were performed in compliance with the Guide for the Care and Use of Laboratory Animals (National Academies Press, 2011) and were approved by the Institutional Biomedical Research Ethics Committee of the Shanghai Institutes for Biological Sciences, Chinese Academy of Sciences. All cell lines are available at ATCC or the Cell Bank, Shanghai Institutes for Biological Sciences, Chinese Academy of Sciences.

\section{Author contributions}

JQ conceived and designed the experimental approach, and JQ, WX, and DG prepared the manuscript as senior authors. YD, NL, and BD performed most experiments. NL contributed to the computational statistical analysis. BD performed the TMA and pathology analyses. WG, HW, QC, HY, YH, HC, SK, XW, PW, TQ, and $\mathrm{QP}$ performed a specific subset of the experiments and analyses, which were supervised by WX, DG, QL, and CP. 


\section{Acknowledgments}

We thank Pierre Chambon for the Brg1-floxed mice. This study was partially supported by the National Key Research and Development Program of China (2017YFA0505500), the Chinese Academy of Sciences (QYZDB-SSW-SMC052), the Strategic Priority Research Program of the Chinese Academy of Sciences (XDB19000000 and XDA12000000), the National Natural Science Foundation of China Distinguished Young Scholars Program (81825018), and the National Natural Science Foundation of China Projects (31471281, 81773121, 81802818, 81772723, 81830054, $81572536,81772742$, and 81672850$)$. JQ is a scholar in the National 1000 Young Talents Program of China, and NL is supported by the Initiative Postdocs Supporting Program by MOHRSS and the National Postdoc Management Committee (bx201800247).

Address correspondence to: Jun Qin (Shanghai Institute of Nutrition and Health Sciences) or Dong Gao (Shanghai Institute of Biochemistry and Cell Biology), Shanghai Institutes for Biological Sciences, Chinese Academy of Sciences, 320 Yueyang Road, Shanghai 200031, China. Phone: 86.21.54923326; Email: qinjun@sibs.ac.cn (JQ). Phone: 86.21.54921117; Email: dong.gao@sibcb.ac.cn (DG). Or to: Wei Xue, Department of Urology, Renji Hospital, School of Medicine, Shanghai Jiaotong University, 160 Pujian Road, Shanghai 200127, China. Phone: 86.21.68385615; Email: xuewei@renji.com.
1. Shen MM, Abate-Shen C. Molecular genetics of prostate cancer: new prospects for old challenges. Genes Dev. 2010;24(18):1967-2000.

2. Robinson $\mathrm{D}$, et al. Integrative clinical genomics of advanced prostate cancer. Cell. 2015;161(5):1215-1228.

3. Taylor BS, et al. Integrative genomic profiling of human prostate cancer. Cancer Cell. 2010;18(1):11-22.

4. Tomlins SA, et al. Recurrent fusion of TMPRSS2 and ETS transcription factor genes in prostate cancer. Science. 2005;310(5748):644-648.

5. Barbieri CE, et al. Exome sequencing identifies recurrent SPOP, FOXA1 and MED12 mutations in prostate cancer. Nat Genet. 2012;44(6):685-689.

6. Wedge DC, et al. Sequencing of prostate cancers identifies new cancer genes, routes of progression and drug targets. Nat Genet. 2018;50(5):682-692.

7. Armenia J, et al. The long tail of oncogenic drivers in prostate cancer. Nat Genet. 2018;50(5):645-651.

8. Wang S, et al. Prostate-specific deletion of the murine Pten tumor suppressor gene leads to metastatic prostate cancer. Cancer Cell. 2003;4(3):209-221

9. Trotman LC, et al. Pten dose dictates cancer progression in the prostate. PLoS Biol. 2003;1(3):E59.

10. Chen Z, et al. Crucial role of p53-dependent cellular senescence in suppression of Pten-deficient tumorigenesis. Nature. 2005;436(7051):725-730.

11. Carver BS, et al. Aberrant ERG expression cooperates with loss of PTEN to promote cancer progression in the prostate. Nat Genet. 2009;41(5):619-624.

12. King JC, et al. Cooperativity of TMPRSS2-ERG with PI3-kinase pathway activation in prostate oncogenesis. Nat Genet. 2009;41(5):524-526.

13. Mulholland DJ, et al. Pten loss and RAS/MAPK activation cooperate to promote EMT and metastasis initiated from prostate cancer stem/progenitor cells. Cancer Res. 2012;72(7):1878-1889.

14. Abate-Shen C, et al. Nkx3.1; Pten mutant mice develop invasive prostate adenocarcinoma and lymph node metastases. Cancer Res. 2003;63(14):3886-3890.

15. O'Neil NJ, Bailey ML, Hieter P. Synthetic lethality and cancer. Nat Rev Genet. 2017;18(10):613-623.

16. Farmer H, et al. Targeting the DNA repair defect in BRCA mutant cells as a therapeutic strategy. Nature. 2005;434(7035):917-921.

17. Fong PC, et al. Inhibition of poly(ADP-ribose) polymerase in tumors from BRCA mutation car- riers. $N$ Engl J Med. 2009;361(2):123-134.

18. Flavahan WA, Gaskell E, Bernstein BE. Epigenetic plasticity and the hallmarks of cancer. Science. 2017;357(6348):eaal2380.

19. Kim KH, Roberts CW. Targeting EZH2 in cancer. Nat Med. 2016;22(2):128-134.

20. Yuan H, et al. Histone methyltransferase SETD2 modulates alternative splicing to inhibit intestinal tumorigenesis. JClin Invest. 2017;127(9):3375-3391.

21. Bitler BG, et al. Synthetic lethality by targeting EZH2 methyltransferase activity in ARID1Amutated cancers. Nat Med. 2015;21(3):231-238.

22. $\mathrm{Kim} \mathrm{KH}$, et al. SWI/SNF-mutant cancers depend on catalytic and non-catalytic activity of EZH2. Nat Med. 2015;21(12):1491-1496.

23. Helming KC, et al. ARID1B is a specific vulnerability in ARID1A-mutant cancers. Nat Med. 2014;20(3):251-254.

24. Zhao D, et al. Synthetic essentiality of chromatin remodelling factor CHD1 in PTEN-deficient cancer. Nature. 2017;542(7642):484-488.

25. Helming KC, Wang X, Roberts CWM. Vulnerabilities of mutant SWI/SNF complexes in cancer. Cancer Cell. 2014;26(3):309-317.

26. Reisman DN, Sciarrotta J, Wang W, Funkhouser WK, Weissman BE. Loss of BRG1/BRM in human lung cancer cell lines and primary lung cancers: correlation with poor prognosis. Cancer Res. 2003;63(3):560-566.

27. Jelinic P, et al. Recurrent SMARCA4 mutations in small cell carcinoma of the ovary. Nat Genet. 2014;46(5):424-426.

28. Wilson BG, Roberts CW. SWI/SNF nucleosome remodellers and cancer. Nat Rev Cancer. 2011;11(7):481-492.

29. Roy N, et al. Brg1 promotes both tumor-suppressive and oncogenic activities at distinct stages of pancreatic cancer formation. Genes Dev. 2015;29(6):658-671.

30. Holik AZ, et al. Brg1 loss attenuates aberrant wnt-signalling and prevents wnt-dependent tumourigenesis in the murine small intestine. PLoS Genet. 2014;10(7):e1004453.

31. von Figura $G$, et al. The chromatin regulator Brg1 suppresses formation of intraductal papillary mucinous neoplasm and pancreatic ductal adenocarcinoma. Nat Cell Biol. 2014;16(3):255-267.

32. Xu F, et al. Decreased TSPAN1 promotes prostate cancer progression and is a marker for early biochemical recurrence after radical prostatectomy. Oncotarget. 2016;7(39):63294-63305.
33. Li N, et al. AKT-mediated stabilization of histone methyltransferase WHSC1 promotes prostate cancer metastasis. JClin Invest. 2017;127(4):1284-1302.

34. Sumi-Ichinose C, Ichinose H, Metzger D, Chambon P. SNF2beta-BRG1 is essential for the viability of F9 murine embryonal carcinoma cells. Mol Cell Biol. 1997;17(10):5976-5986.

35. Drost J, et al. Organoid culture systems for prostate epithelial and cancer tissue. Nat Protoc. 2016;11(2):347-358.

36. Gao D, et al. Organoid cultures derived from patients with advanced prostate cancer. Cell. 2014;159(1):176-187.

37. Ellwood-Yen K, et al. Myc-driven murine prostate cancer shares molecular features with human prostate tumors. Cancer Cell. 2003;4(3):223-238.

38. Gao D, Inuzuka H, Tseng A, Chin RY, Toker A, Wei W. Phosphorylation by Akt1 promotes cytoplasmic localization of Skp2 and impairs APCCdh1-mediated Skp2 destruction. Nat Cell Biol. 2009;11(4):397-408.

39. Stambolic V, Woodgett JR. Mitogen inactivation of glycogen synthase kinase-3 beta in intact cells via serine 9 phosphorylation. Biochem J. 1994;303 (Pt 3):701-704.

40. Cross DA, Alessi DR, Cohen P, Andjelkovich M, Hemmings BA. Inhibition of glycogen synthase kinase- 3 by insulin mediated by protein kinase B. Nature. 1995;378(6559):785-789.

41. Cohen P, Frame S. The renaissance of GSK3. Nat Rev Mol Cell Biol. 2001;2(10):769-776.

42. Hart M, et al. The F-box protein beta-TrCP associates with phosphorylated beta-catenin and regulates its activity in the cell. Curr Biol. 1999;9(4):207-210.

43. Nakayama KI, Nakayama K. Ubiquitin ligases: cell-cycle control and cancer. Nat Rev Cancer. 2006;6(5):369-381.

44. Jackson PK, Eldridge AG. The SCF ubiquitin ligase: an extended look. Mol Cell. 2002;9(5):923-925.

45. Creyghton MP, et al. Histone H3K27ac separates active from poised enhancers and predicts developmental state. Proc Natl Acad Sci US A. 2010;107(50):21931-21936.

46. Feinberg AP, Koldobskiy MA, Göndör A. Epigenetic modulators, modifiers and mediators in cancer aetiology and progression. Nat Rev Genet. 2016;17(5):284-299.

47. Dai X, et al. Prostate cancer-associated SPOP mutations confer resistance to BET inhibitors through stabilization of BRD4. Nat Med. 
2017;23(9):1063-1071.

48. Cha TL, et al. Akt-mediated phosphorylation of EZH2 suppresses methylation of lysine 27 in histone H3. Science. 2005;310(5746):306-310.

49. Dang CV. MYC on the path to cancer. Cell. 2012;149(1):22-35.

50. Qin J, et al. Androgen deprivation-induced $\mathrm{NCoA} 2$ promotes metastatic and castration-resistant prostate cancer. J Clin Invest. 2014;124(11):5013-5026.

51. Lukacs RU, Memarzadeh S, Wu H, Witte ON. Bmi- 1 is a crucial regulator of prostate stem cell self-renewal and malignant transformation. Cell Stem Cell. 2010;7(6):682-693.

52. Bultman S, et al. A Brg1 null mutation in the mouse reveals functional differences among mammalian SWI/SNF complexes. Mol Cell. 2000;6(6):1287-1295.

53. Reyes JC, Barra J, Muchardt C, Camus A, Babinet C, Yaniv M. Altered control of cellular proliferation in the absence of mammalian brahma (SNF2alpha). EMBO J. 1998;17(23):6979-6991. 54. Li W, et al. MAGeCK enables robust identifi- cation of essential genes from genome-scale CRISPR/Cas9 knockout screens. Genome Biol. 2014;15(12):554.

55. Li W, et al. Quality control, modeling, and visualization of CRISPR screens with MAGeCK-VISPR. Genome Biol. 2015;16:281.

56. Lotan TL, et al. PTEN protein loss by immunostaining: analytic validation and prognostic indicator for a high risk surgical cohort of prostate cancer patients. Clin Cancer Res. 2011;17(20):6563-6573.

57. Jin C, McKeehan K, Wang F. Transgenic mouse with high Cre recombinase activity in all prostate lobes, seminal vesicle, and ductus deferens. Prostate. 2003;57(2):160-164.

58. Gao D, et al. A Tmprss2-CreERT2 knock-in mouse model for cancer genetic studies on prostate and colon. PLOS ONE. 2016;11(8):e0161084.

59. Park JH, et al. Prostatic intraepithelial neoplasia in genetically engineered mice. Am J Pathol. 2002;161(2):727-735.

60. Qin J, et al. COUP-TFII inhibits TGF- $\beta$-induced growth barrier to promote prostate tumorigene- sis. Nature. 2013;493(7431):236-240.

61. Audic S, Claverie JM. The significance of digital gene expression profiles. Genome Res. 1997;7(10):986-995.

62. Olsen MB, et al. NEIL3-dependent regulation of cardiac fibroblast proliferation prevents myocardial rupture. Cell Rep. 2017;18(1):82-92.

63. You J, et al. Regulatory protein SrpA controls phage infection and core cellular processes in Pseudomonas aeruginosa. Nat Commun. 2018;9(1):1846.

64. Xu J, et al. Nuclear carbonic anhydrase $6 \mathrm{~B}$ associates with PRMT5 to epigenetically promote IL-12 expression in innate response. Proc Natl Acad Sci US A. 2017;114(32):8620-8625.

65. Shin H, Liu T, Manrai AK, Liu XS. CEAS: cis-regulatory element annotation system. Bioinformatics. 2009;25(19):2605-2606.

66. Ramírez F, et al. deepTools2: a next generation web server for deep-sequencing data analysis. Nucleic Acids Res. 2016;44(W1):W160-W165.

67. Ding Z, et al. SMAD4-dependent barrier constrains prostate cancer growth and metastatic progression. Nature. 2011;470(7333):269-273. 